\title{
Bio-Derived Catalysts: A Current Trend of Catalysts Used in Biodiesel Production
}

\author{
Hoang Chinh Nguyen ${ }^{1, *,+}$, My-Linh Nguyen ${ }^{2,3,+}$, Chia-Hung Su ${ }^{3, *}$, Hwai Chyuan Ong ${ }^{4, *}$, Horng-Yi Juan ${ }^{3}$ \\ and Shao-Jung $\mathrm{Wu}^{3}$
}

1 Faculty of Applied Sciences, Ton Duc Thang University, Ho Chi Minh City 700000, Vietnam

2 Graduate Institute of Applied Science and Technology, National Taiwan University of Science and Technology, Taipei City 106335, Taiwan; nguyenmylinh011293@gmail.com

3 Department of Chemical Engineering, Ming Chi University of Technology, New Taipei City 243303, Taiwan; hyjuan@mail.mcut.edu.tw (H.-Y.J.); sjwu@mail.mcut.edu.tw (S.-J.W.)

4 Centre for Green Technology, Faculty of Engineering and Information Technology, University of Technology Sydney, Ultimo, NSW 2007, Australia

* Correspondence: nguyenhoangchinh@tdtu.edu.vn (H.C.N.); chsu@mail.mcut.edu.tw (C.-H.S.); HwaiChyuan.Ong@uts.edu.au (H.C.O.); Tel.: +84-28-37755058 (H.C.N.); +886-229089899 (C.-H.S.); $+61-433763110$ (H.C.O.)

+ These authors contributed equally to this work.

check for updates

Citation: Nguyen, H.C.; Nguyen, M.-L.; Su, C.-H.; Ong, H.C.; Juan, H.-Y.; Wu, S.-J. Bio-Derived Catalysts: A Current Trend of Catalysts Used in Biodiesel Production. Catalysts 2021, 11, 812. https://doi.org/10.3390/ catal11070812

Academic Editor: Gartzen Lopez

Received: 3 June 2021

Accepted: 29 June 2021

Published: 1 July 2021

Publisher's Note: MDPI stays neutral with regard to jurisdictional claims in published maps and institutional affiliations.

Copyright: (c) 2021 by the authors. Licensee MDPI, Basel, Switzerland. This article is an open access article distributed under the terms and conditions of the Creative Commons Attribution (CC BY) license (https:// creativecommons.org/licenses/by/ $4.0 /)$
Abstract: Biodiesel is a promising alternative to fossil fuels and mainly produced from oils/fat through the (trans)esterification process. To enhance the reaction efficiency and simplify the production process, various catalysts have been introduced for biodiesel synthesis. Recently, the use of bio-derived catalysts has attracted more interest due to their high catalytic activity and ecofriendly properties. These catalysts include alkali catalysts, acid catalysts, and enzymes (biocatalysts), which are (bio)synthesized from various natural sources. This review summarizes the latest findings on these bio-derived catalysts, as well as their source and catalytic activity. The advantages and disadvantages of these catalysts are also discussed. These bio-based catalysts show a promising future and can be further used as a renewable catalyst for sustainable biodiesel production.

Keywords: alternative fuel; bio-derived catalyst; biodiesel; ecofriendly benefit; (trans)esterification

\section{Introduction}

Global industrialization leads to the extensive use of fuel-based energy for transportation, which consequently causes the depletion of fossil fuels and global warming. Therefore, renewable fuels are considered an alternative energy to solve the problem of fuel depletion and environmental pollution. Biodiesel, a biomass-derived fuel, is a promising bioenergy, which is increasingly produced worldwide to replace fossil fuels because of its combustion efficiency, compatibility with diesel engines, and low carbon dioxide emissions [1,2]. As a result, studies have been focusing on developing an efficient approach for biodiesel production.

Biodiesel is mainly synthesized from oils/fat (derived from plants, animals, and microorganisms) through the (trans)esterification process [3,4]. The efficiency of this process is mainly affected by several factors including the quality of feedstock (level of free fatty acids), the type of acyl acceptor (e.g., alcohols or methyl acetate), and the type of reactions (e.g., noncatalytic reaction, chemical-catalyzed reaction, and enzymatic reaction) $[5,6]$. To enhance the reaction efficiency, most studies focused on developing efficient catalysts for the (trans)esterification reaction. Consequently, different types of catalysts, such as alkali catalysts, acid catalysts, and enzymes have been studied [7,8]. Commonly, chemical catalysts (such as $\mathrm{KOH}, \mathrm{NaOH}, \mathrm{H}_{2} \mathrm{SO}_{4}$, and $\mathrm{HCl}$ ) are used for biodiesel production processes [9]. Although these chemical catalysts efficiently catalyze the reaction, they retain several limitations regarding their reusability, negative effect on the environment, and 
complicated purification steps in the downstream process [2]. Therefore, studies have shifted to using bio-derived catalysts for biodiesel production.

Bio-based catalysts have increasingly attracted attention for biodiesel production due to their availability and environmentally friendly nature [8]. Those catalysts are derived from natural sources and divided into 3 types: alkali catalysts, acid catalysts, and biocatalysts (enzymes). Each type of catalyst has its advantages and disadvantages for biodiesel production. To synthesize these catalysts, various biomass materials and synthesis methods have been reported [8]. Catalysts derived from different sources possess different catalytic activity. This review aims to summarize the bio-derived catalysts for biodiesel production. The natural sources used for catalyst synthesis are reported. The advantages and disadvantages of each type of catalyst are also discussed in this review.

\section{Bio-Derived Alkali Catalysts for Biodiesel Production}

Biodiesel is commonly produced through transesterification of vegetable oil or animal fat with short-chain alcohols (e.g., methanol and ethanol) in the presence of liquid/homogenous alkali catalysts (e.g., $\mathrm{NaOH}, \mathrm{KOH}$ ). The homogenous alkali catalystcatalyzed transesterifications achieve high biodiesel yield within a short reaction time (30-45 $\mathrm{min}$ ) and proceed at atmospheric pressure [10]. However, emulsification, difficulty in separation of the catalyst after reaction, and generation of excess wastewater are major problems associated with those catalysts [11]. To address these issues, heterogeneous alkali catalysts have been increasingly developed as alternative catalysts for biodiesel production. The use of heterogeneous alkali catalysts for biodiesel production simplifies the operation, easily removes and recovers catalysts from the reaction mixture, results in better biodiesel refining, and lowers environmental problems as compared to homogenous catalysts [10]. Furthermore, heterogeneous alkali catalysts can be synthesized from various cheap materials, thus reducing the production cost [12]. However, heterogeneous catalytic reactions are typically time-consuming and require a higher reaction temperature than the homogenous alkali catalyst-catalyzed transesterification due to diffusion problems owing to the formation of three phases of the reactants (methanol-oil-solid catalyst) [8]. Therefore, studies have focused on developing solid catalysts with high catalytic activity to produce biodiesel under mild reaction conditions and short reaction time [13].

Alkali catalysts derived from biomass have attracted considerable interest in biodiesel production due to their ecofriendly nature, low cost, and the availability of biomass as a material for the synthesis of catalysts. Moreover, the use of biomass for catalyst synthesis can solve the environmental problems caused by surplus biomass waste. Therefore, various types of bio-derived alkali catalysts have been studied for transesterification reactions. Biomass-derived calcium oxide $(\mathrm{CaO})$ is one of the most promising solid alkali catalysts used for biodiesel production [14]. The availability of $\mathrm{CaO}$ has been recorded in different types of waste/low-cost materials, mainly from animal-derived biomass, including eggshell [15], Turbo jourdani shell [16], oyster shell [17], Pomacea canaliculata shell [18], Turbonilla striatula shell [19], crap shell [20], mussel (Perna varidis) shell [21], Grooved razor shell [22], conch shell [23], Malleus malleus shells [24], and animal bone [25]. The catalytic activities of the synthesized $\mathrm{CaO}$ catalysts vary, depending on the materials used and the synthesis method. Among the materials used, eggshell seems to be one of the most suitable materials and attracted extensive investigations for the synthesis of $\mathrm{CaO}$ catalyst since it contains a high level of $\mathrm{CaCO}_{3}$ and is easy to obtain [26]. Yaşar [26] reported the synthesis of a $\mathrm{CaO}$ catalyst from waste eggshell. The transesterification reaction catalyzed by eggshell-derived $\mathrm{CaO}$ resulted in $96.81 \%$ biodiesel yield, compared to $95.12 \%$ biodiesel produced by commercial $\mathrm{CaO}$, under the reaction conditions of $4 \%$ catalysts, $1 \mathrm{~h}$ reaction time, and $60^{\circ} \mathrm{C}$ reaction temperature [26]. The synthesis method also seems to affect the catalytic activity of the catalyst; therefore, catalysts are derived from the same materials, but they exhibit various catalytic activities (yielding 90-97\% biodiesel) [26,27]. For example, Gollakota et al. [28] used eggshell-supported pyrolysis residue as a solid alkali catalyst for transesterification of waste cooking oil (WCO). This study also compared the catalytic 
activity of unsupported eggshell catalysts and the supported catalyst. Results revealed that the biodiesel yield reached over $95 \%$ at $65^{\circ} \mathrm{C}$ using $10 \%$ supported catalyst with a methanol to oil molar ratio of 12:1 in $3 \mathrm{~h}$. In comparison with unsupported eggshell catalysts, the synthesized catalyst shows improved surface area and catalytic activity [28]. Goli et al. [29] reported biodiesel production from soybean oil using a $\mathrm{CaO}$ catalyst that was derived from chicken eggshell waste, yielding 93\% biodiesel, whereas Kirubakaran et al. [27] also used a waste chicken eggshell-derived $\mathrm{CaO}$ catalyst for biodiesel production and reported $90.41 \%$ biodiesel yield under optimal reaction conditions.

In addition to $\mathrm{CaO}$, other calcium (Ca)-based catalysts synthesized from biomass have been reported as potential alkali catalysts for biodiesel production [15,30]. Gupta et al. [15] synthesized eggshell-CaDG catalyst for biodiesel production. The transesterification of WCO was conducted to compare the catalytic activity of eggshell-CaOC-H-D and eggshellCaDG. Under optimized reaction conditions (catalyst loading of $1.5 \%$, reaction time of $50 \mathrm{~min}$, methanol:oil molar ratio of $10: 1$, temperature of $60^{\circ} \mathrm{C}$, and agitation speed of $300 \mathrm{rpm}$ ), the eggshell-CaDG catalyzed reaction provided $96.07 \%$ biodiesel. The eggshellCaDG demonstrated higher catalytic activity than eggshell-CaOC-H-D ( $93.10 \%$ biodiesel yield under the optimal reaction conditions of temperature of $65^{\circ} \mathrm{C}$, catalyst loading of $3 \%$, methanol:oil molar ratio of 12:1, $400 \mathrm{rpm}$, and reaction time of $90 \mathrm{~min}$ ) [15]. Both catalysts (eggshell-CaOC-H-D and eggshell-CaDG) could be reused for up to five cycles for biodiesel production [15]. In addition, alkali catalyst obtained from the plant materials through calcination method such as calcined Musa acuminata peduncle [30], calcined waste cupuaçu (Theobroma grandiflorum) seeds [31], calcined banana peel [32], calcined elephantear tree pod husk [33], calcined kola nut husk pod [34], calcined Brassica nigra plant [35], $\mathrm{ZrO}_{2}$-supported bamboo leaf ash [36], calcined Sesamum indicum ash [37], calcined Tectona grandis leaves [38], calcined fig (Ficus carica) leaves [39], calcined ginger (Zingiber officinale) leaves [40], calcinated Carica Papaya stem [41], and calcined Musa balbisiana Colla peel [42], also efficiently converted oil into biodiesel with conversion rates of higher than $95.1 \%$ (Table 1).

Activated carbon-based and biochar catalysts derived from biomass are another type of alkali catalyst, which shows promise for biodiesel production. These catalysts are mainly synthesized from plant materials through the carbonization process. Recently, Naeem et al. [43] reported the use of $\mathrm{KOH} /$ corn cob activated carbon catalyst for biodiesel production with a biodiesel yield of $97.8 \%$, whereas the nano-bifunctional catalyst from rice husk resulted in $98.6 \%$ biodiesel yield [44]. Due to their high catalytic activity, the synthesis of these bio-based activated carbon catalysts and other bio-based alkali catalysts is still an objective of investigation for biodiesel production.

Table 1. Several bio-derived alkali catalysts for biodiesel production.

\begin{tabular}{|c|c|c|c|c|c|c|c|c|}
\hline \multirow[b]{2}{*}{ Catalyst } & \multirow[b]{2}{*}{ Feedstock } & \multicolumn{4}{|c|}{ Reaction Conditions } & \multirow[b]{2}{*}{$\begin{array}{l}\text { Conversion/ } \\
\text { Biodiesel } \\
\text { Yield (\%) }\end{array}$} & \multirow[b]{2}{*}{$\begin{array}{c}\text { Time of } \\
\text { Reuse/Corresponding } \\
\text { Biodiesel Yield (\%) }\end{array}$} & \multirow[b]{2}{*}{ Ref. } \\
\hline & & $\begin{array}{c}\text { Catalyst } \\
\text { Loading (\%) }\end{array}$ & $\begin{array}{c}\text { Alcohol: } \\
\text { Fatty Acid } \\
\text { Molar Ratio }\end{array}$ & $\begin{array}{l}\text { Time } \\
\text { (min) }\end{array}$ & $\begin{array}{l}\text { Temp. } \\
\left({ }^{\circ} \mathrm{C}\right)\end{array}$ & & & \\
\hline $\begin{array}{l}\text { Eggshell-derived } \\
\mathrm{CaO}\end{array}$ & Rapeseed oil & 4 & 9:1 & 60 & 60 & 95.12 & $3 / 93.24$ & [12] \\
\hline $\begin{array}{c}\text { Chicken } \\
\text { eggshell-derived } \\
\mathrm{CaO}\end{array}$ & WCO & 1.5 & 10:1 & 50 & 60 & 96.07 & $5 / 81.15$ & [15] \\
\hline $\begin{array}{c}\text { Chicken } \\
\text { eggshell-derived } \\
\mathrm{CaO}\end{array}$ & Chicken fat & 8.5 & 13:1 & 300 & 57.5 & 90.41 & $5 / 85$ & [27] \\
\hline $\begin{array}{c}\text { Chicken } \\
\text { eggshell-derived } \\
\mathrm{CaO}\end{array}$ & WCO & 10 & $12: 1$ & 180 & 65 & 95 & - & [28] \\
\hline
\end{tabular}


Table 1. Cont.

\begin{tabular}{|c|c|c|c|c|c|c|c|c|}
\hline \multirow[b]{2}{*}{ Catalyst } & \multirow[b]{2}{*}{ Feedstock } & \multicolumn{4}{|c|}{ Reaction Conditions } & \multirow[b]{2}{*}{$\begin{array}{l}\text { Conversion/ } \\
\text { Biodiesel } \\
\text { Yield (\%) }\end{array}$} & \multirow[b]{2}{*}{$\begin{array}{c}\text { Time of } \\
\text { Reuse/Corresponding } \\
\text { Biodiesel Yield (\%) }\end{array}$} & \multirow[b]{2}{*}{ Ref. } \\
\hline & & $\begin{array}{c}\text { Catalyst } \\
\text { Loading (\%) }\end{array}$ & $\begin{array}{c}\text { Alcohol: } \\
\text { Fatty Acid } \\
\text { Molar Ratio }\end{array}$ & $\begin{array}{l}\text { Time } \\
(\mathrm{min})\end{array}$ & $\begin{array}{l}\text { Temp. } \\
\left({ }^{\circ} \mathrm{C}\right)\end{array}$ & & & \\
\hline $\begin{array}{c}\text { Chicken } \\
\text { eggshell-derived } \\
\mathrm{CaO}\end{array}$ & Soybean oil & 7 & 10:1 & 180 & 57.5 & 93 & $4 / 75$ & [29] \\
\hline $\begin{array}{c}\text { Eggshell-derived } \\
\mathrm{CaO}\end{array}$ & $\begin{array}{l}\text { Phoenix dactylifera } \\
\text { L. seed oil }\end{array}$ & 5 & $12: 1$ & 90 & 65 & 93.5 & $6 />80$ & [45] \\
\hline $\begin{array}{c}\text { Eggshell-derived } \\
\mathrm{CaO} / \mathrm{SiO}_{2}\end{array}$ & WCO & 8 & $14: 1$ & 60 & 60 & 91 & $10 />85$ & [46] \\
\hline $\begin{array}{c}\text { Chicken } \\
\text { eggshell-derived } \\
\mathrm{CaO}\end{array}$ & Jatropha curcas oil & 2 & $6: 1$ & 120 & 90 & 98 & - & [47] \\
\hline $\begin{array}{c}\text { Chicken } \\
\text { eggshell-derived } \\
\mathrm{CaO}\end{array}$ & $\begin{array}{l}\text { Chlorella vulgaris } \\
\text { biomass }\end{array}$ & 1.39 & 10:1 & 180 & 70 & 92.03 & $3 />85.2$ & [48] \\
\hline $\begin{array}{c}\mathrm{Fe}_{3} \mathrm{O}_{4} \\
\text { nanoparticles } \\
\text { impregnated } \\
\text { eggshell }\end{array}$ & $\begin{array}{l}\text { Pongamia pinnata } \\
\text { oil }\end{array}$ & 2 & $12: 1$ & 120 & 65 & 98 & $7 / 98$ & [49] \\
\hline $\begin{array}{c}\text { Chicken } \\
\text { eggshell-derived } \\
\mathrm{CaO}\end{array}$ & $\begin{array}{l}\text { Terminalia belleric } \\
\text { seed oil }\end{array}$ & 2.25 & $9: 1$ & 90 & 62.5 & 97.98 & - & [50] \\
\hline $\begin{array}{c}\text { Chicken } \\
\text { eggshell-derived } \\
\mathrm{CaO}\end{array}$ & Palm kernel oil & 4 & 10:1 & 60 & 50 & 97.1 & $5 />90$ & [51] \\
\hline $\begin{array}{c}\mathrm{Al}_{2} \mathrm{O}_{3} \text { impregnated } \\
\text { on calcined } \\
\text { eggshells }\end{array}$ & Rubber seed oil & 3 & $12: 1$ & 240 & 65 & 98.9 & - & [52] \\
\hline $\begin{array}{c}\text { Chicken } \\
\text { eggshell-derived } \\
\mathrm{CaO}\end{array}$ & Rubber seed oil & 5 & $9: 1$ & 240 & 65 & 97.84 & - & [53] \\
\hline $\begin{array}{l}\text { Eggshell-derived } \\
\text { CaO supported on } \\
\text { a fly ash-based } \\
\text { zeolitic material }\end{array}$ & Sunflower oil & 6 & $6: 1$ & 30 & 60 & 99.2 & $5 / 97.9$ & [54] \\
\hline $\begin{array}{c}\text { Chicken } \\
\text { eggshell-derived } \\
\mathrm{CaO}\end{array}$ & WCO & 5 & $9: 1$ & 165 & 65 & 87.8 & - & [55] \\
\hline $\begin{array}{c}\text { Palm mill fly } \\
\text { ash-supported } \mathrm{CaO} \\
\text { derived from } \\
\text { eggshells } \\
\text { (CaO/PMFA) }\end{array}$ & Palm oil & 6 & 10:1 & 180 & 70 & 86.2 & $5 / 70$ & [56] \\
\hline $\begin{array}{l}\text { KOH impregnated } \\
\text { eggshell }\end{array}$ & $\begin{array}{l}\text { Reutealis } \\
\text { trisperma oil }\end{array}$ & 5 & $12: 1$ & 60 & 60 & 94 & - & [57] \\
\hline $\begin{array}{l}\text { Eggshell-derived } \\
\text { CaO supported } \\
\text { W-Mo mixed oxide }\end{array}$ & WCO & 2 & $15: 1$ & 120 & 70 & 96.2 & $5 / 90$ & [58] \\
\hline $\begin{array}{c}\mathrm{KF} / \text { eggshell- } \\
\mathrm{Fe}_{3} \mathrm{O}_{4}\end{array}$ & Neem oil & 6 & $15: 1$ & 120 & 65 & 97 & $5 />75$ & [59] \\
\hline $\begin{array}{c}\text { Chicken } \\
\text { eggshell-derived } \\
\mathrm{CaO}\end{array}$ & Sunflower oil & 5 & 11:1 & 180 & 60 & 83.2 & $4 />80$ & [60] \\
\hline
\end{tabular}


Table 1. Cont.

\begin{tabular}{|c|c|c|c|c|c|c|c|c|}
\hline \multirow[b]{2}{*}{ Catalyst } & \multirow[b]{2}{*}{ Feedstock } & \multicolumn{4}{|c|}{ Reaction Conditions } & \multirow[b]{2}{*}{$\begin{array}{l}\text { Conversion/ } \\
\text { Biodiesel } \\
\text { Yield (\%) }\end{array}$} & \multirow{2}{*}{$\begin{array}{c}\text { Time of } \\
\text { Reuse/Corresponding } \\
\text { Biodiesel Yield (\%) }\end{array}$} & \multirow[b]{2}{*}{ Ref. } \\
\hline & & $\begin{array}{c}\text { Catalyst } \\
\text { Loading (\%) }\end{array}$ & $\begin{array}{c}\text { Alcohol: } \\
\text { Fatty Acid } \\
\text { Molar Ratio }\end{array}$ & $\begin{array}{l}\text { Time } \\
(\min )\end{array}$ & $\begin{array}{l}\text { Temp. } \\
\left({ }^{\circ} \mathrm{C}\right)\end{array}$ & & & \\
\hline $\begin{array}{c}\mathrm{La}_{2} \mathrm{O}_{3} / \mathrm{CaO} \\
\text { derived from } \\
\text { eggshell }\end{array}$ & Palm oil & 10 & $12: 1$ & 250 & 60 & 92.3 & - & [61] \\
\hline $\begin{array}{c}\text { Ostrich } \\
\text { eggshell-derived } \\
\mathrm{CaO}\end{array}$ & WCO & 1.57 & $11: 1$ & 114 & 65 & 97.54 & - & [62] \\
\hline $\begin{array}{c}\text { Chicken } \\
\text { eggshell-derived } \\
\mathrm{CaO}\end{array}$ & WCO & 1.61 & 11.4:1 & 114 & 65 & 94.7 & - & [62] \\
\hline $\begin{array}{c}\mathrm{Fe}_{3} \mathrm{O}_{4} / \mathrm{CaO} \\
\text { derived from } \\
\text { eggshell }\end{array}$ & Palm oil & 6 & $10: 1$ & 120 & 70 & 90 & - & [63] \\
\hline $\begin{array}{c}\text { Chicken } \\
\text { eggshell-derived } \\
\mathrm{CaO}\end{array}$ & WCO & 1.47 & $7.85: 1$ & 144 & 43 & 90.13 & $3 / 73.3$ & [64] \\
\hline $\begin{array}{l}\text { Eggshells-derived } \\
\mathrm{CaO}\end{array}$ & Rubber seed oil & 4 & $12: 1$ & 180 & 65 & 99.6 & $7 / 86.4$ & [65] \\
\hline $\begin{array}{c}\text { Chicken } \\
\text { eggshell-derived } \\
\mathrm{CaO}\end{array}$ & Soybean oil & 3 & $9: 1$ & 240 & 65 & 94.2 & - & [66] \\
\hline $\begin{array}{c}\text { Quail } \\
\text { eggshell-derived } \\
\mathrm{CaO}\end{array}$ & Soybean oil & 3 & $9: 1$ & 240 & 65 & 94.8 & - & [66] \\
\hline $\begin{array}{c}\mathrm{CaO} @ \mathrm{MgO} \\
\text { nanocatalyst } \\
\text { derived from } \\
\text { chicken eggshell }\end{array}$ & Waste edible oil & 4.571 & $16.7: 1$ & 424.8 & 69.37 & 98.37 & - & [67] \\
\hline $\begin{array}{l}\mathrm{SrO} / \mathrm{CaO} \text { derived } \\
\text { from eggshell }\end{array}$ & Jatropha oil & 4.77 & $27.6: 1$ & 89.8 & 65 & 99.71 & $5 />60 \%$ & [68] \\
\hline $\begin{array}{l}\text { Na-K doped } \mathrm{CaO} \\
\text { derived from } \\
\text { calcined eggshell } \\
\left(\mathrm{Na}_{1} \mathrm{~K}_{1} / \mathrm{CaO}\right)\end{array}$ & Canola oil & 3 & $9: 1$ & 180 & 50 & 97.6 & $4 / 66.0$ & [69] \\
\hline $\begin{array}{l}\text { Egg shell-derived } \\
\text { nano-CaO }\end{array}$ & $\begin{array}{c}\text { Chlorella } \\
\text { pyrenoidosa oil }\end{array}$ & 2.06 & $30: 1$ & 180 & 60 & 93.44 & $6 / 85.2$ & [70] \\
\hline $\begin{array}{c}\text { Duck } \\
\text { eggshell-derived } \\
\mathrm{CaO}\end{array}$ & $\begin{array}{l}\text { Momordica } \\
\text { charantia oil }\end{array}$ & 10 & & 80 & 65 & 96.8 & - & [71] \\
\hline $\begin{array}{l}\text { Na impregnated } \\
\text { calcined eggshell }\end{array}$ & $\begin{array}{l}\text { Madhuca indica } \\
\text { oil }\end{array}$ & 5 & $9: 1$ & 60 & 60 & 81.56 & $5 />70$ & [72] \\
\hline $\begin{array}{c}\text { Zn doped } \\
\text { eggshell-derived } \\
\mathrm{CaO}\end{array}$ & WCO & 5 & $20: 1$ & 240 & 65 & 96.74 & $5 / 64.5$ & [73] \\
\hline $\begin{array}{c}\text { Zn doped } \\
\text { eggshell-derived } \\
\mathrm{CaO}\end{array}$ & Eucalyptus oil & 5 & $6: 1$ & 150 & 65 & 93.8 & $5 />88$ & [74] \\
\hline $\begin{array}{c}\text { Chicken } \\
\text { eggshell-derived } \\
\mathrm{CaO}\end{array}$ & WCO & 1.5 & $10: 1$ & 210 & 50 & 91.42 & $5 / 48$ & [75] \\
\hline $\begin{array}{c}\text { Chicken } \\
\text { bone-derived } \mathrm{CaO}\end{array}$ & Algal oil & 5 & $9: 1$ & 180 & 65 & 95 & $4 />80$ & [75] \\
\hline
\end{tabular}


Table 1. Cont.

\begin{tabular}{|c|c|c|c|c|c|c|c|c|}
\hline \multirow[b]{2}{*}{ Catalyst } & \multirow[b]{2}{*}{ Feedstock } & \multicolumn{4}{|c|}{ Reaction Conditions } & \multirow[b]{2}{*}{$\begin{array}{l}\text { Conversion/ } \\
\text { Biodiesel } \\
\text { Yield (\%) }\end{array}$} & \multirow{2}{*}{$\begin{array}{c}\text { Time of } \\
\text { Reuse/Corresponding } \\
\text { Biodiesel Yield (\%) }\end{array}$} & \multirow[b]{2}{*}{ Ref. } \\
\hline & & $\begin{array}{c}\text { Catalyst } \\
\text { Loading (\%) }\end{array}$ & $\begin{array}{c}\text { Alcohol: } \\
\text { Fatty Acid } \\
\text { Molar Ratio }\end{array}$ & $\begin{array}{l}\text { Time } \\
(\mathrm{min})\end{array}$ & $\begin{array}{l}\text { Temp. } \\
\left({ }^{\circ} \mathrm{C}\right)\end{array}$ & & & \\
\hline $\begin{array}{c}\text { Chicken } \\
\text { eggshell-derived } \\
\mathrm{CaO}\end{array}$ & Algal oil & 5 & $9: 1$ & 180 & 65 & 94 & $4 / 70$ & [76] \\
\hline $\begin{array}{l}\text { Chicken } \\
\text { manure-derived } \\
\text { catalyst }\end{array}$ & Algal oil & 5 & $9: 1$ & 180 & 65 & 85 & $4 />60$ & [76] \\
\hline $\begin{array}{c}\text { Chicken } \\
\text { eggshell-derived } \\
\text { Ca-based catalysts }\end{array}$ & $\begin{array}{l}\text { Waste cooking } \\
\text { palm oil }\end{array}$ & 3 & $15: 1$ & 180 & 80 & 90.1 & $3 />70$ & [77] \\
\hline $\begin{array}{c}\text { Turbo jourdani } \\
\text { shell-derived } \mathrm{CaO}\end{array}$ & Palm oil & 10 & $3: 1$ & 420 & 80 & 99.33 & $8 />75$ & [16] \\
\hline $\begin{array}{c}\text { Oyster } \\
\text { shell-derived } \mathrm{CaO}\end{array}$ & WCO & 6 & $9: 1$ & 180 & 65 & 87.3 & - & [17] \\
\hline $\begin{array}{l}\text { Pomacea canaliculata } \\
\text { shell-derived } \mathrm{CaO}\end{array}$ & Palm oil & 0.8 & $12: 1$ & 360 & 65 & 95.2 & $4 / 90.7$ & [18] \\
\hline $\begin{array}{l}\text { Activated carbon } \\
\text { supported CaO } \\
\text { from Turbonilla } \\
\text { striatula shell }\end{array}$ & WCO & 11 & $40: 1$ & 420 & 120 & 96 & $5 / 96$ & [19] \\
\hline $\begin{array}{l}\text { Crap shell-derived } \\
\mathrm{CaO}\end{array}$ & Waste fish oil & 2.5 & $12: 1$ & 90 & 65 & 96.6 & $5 / 80$ & [20] \\
\hline $\begin{array}{c}\mathrm{NaOH} \\
\text { impregnated } \\
\text { activated } \\
\text { carbon/CaO } \\
\text { derived Perna } \\
\text { varidis shell }\end{array}$ & Palm oil & 7.5 & $0.5: 1$ & 180 & 65 & 95.12 & - & [21] \\
\hline $\begin{array}{c}\text { Grooved razor } \\
\text { shell-derived } \mathrm{CaO}\end{array}$ & WCO & 5 & $15: 1$ & 180 & 65 & 94 & $5 / 87$ & [22] \\
\hline $\begin{array}{c}\text { Conch } \\
\text { shell-derived } \mathrm{CaO}\end{array}$ & $\begin{array}{l}\text { Moringa oleifera } \\
\text { oil }\end{array}$ & 8.022 & $8.662: 1$ & 130 & 65 & 97.06 & - & [23] \\
\hline $\begin{array}{c}\text { Malleus malleus } \\
\text { shells derived } \mathrm{CaO}\end{array}$ & WCO & 7.5 & $11.85: 1$ & 86.25 & 65 & 93.81 & - & [24] \\
\hline $\begin{array}{l}\text { Calcined sheep } \\
\text { bone impregnated } \\
\text { fly ash catalyst }\end{array}$ & Mustard oil & 10 & $5.5: 1$ & 360 & 65 & 90.4 & $7 / 80.3$ & [25] \\
\hline \multirow{2}{*}{$\begin{array}{l}\text { Snail shell-derived } \\
\mathrm{CaO} \text { nanocatalyst }\end{array}$} & \multirow{2}{*}{$\begin{array}{c}\text { Scum oil } \\
\text { Hydnocarpus } \\
\text { wightiana oil }\end{array}$} & 0.89 & $12.4: 1$ & 145.15 & 61.6 & 98.93 & $5 />90$ & \multirow{2}{*}{ [78] } \\
\hline & & 0.87 & $12.7: 1$ & 119.68 & 58.6 & 96.93 & - & \\
\hline $\begin{array}{l}\text { Snail shell-derived } \\
\qquad \mathrm{CaO}\end{array}$ & Soybean oil & 6 & $9: 1$ & 210 & 65 & 90 & $5 / 80$ & [79] \\
\hline $\begin{array}{l}\mathrm{KOH} \text { impregnated } \\
\text { snail shell }\end{array}$ & Soybean oil & 6 & $9: 1$ & 210 & 65 & 96 & $5 / 90$ & [79] \\
\hline $\begin{array}{l}\text { Snail shell-derived } \\
\mathrm{CaO}\end{array}$ & Soybean oil & 3 & $6: 1$ & 420 & 28 & 98 & $8 / 90$ & [80] \\
\hline $\begin{array}{c}\text { Quail } \\
\text { eggshell-derived } \\
\mathrm{CaO}\end{array}$ & Sunflower oil & 2 & $10.5: 1$ & 120 & 60 & 99 & $3 / 78.26$ & [81] \\
\hline
\end{tabular}


Table 1. Cont.

\begin{tabular}{|c|c|c|c|c|c|c|c|c|}
\hline \multirow[b]{2}{*}{ Catalyst } & \multirow[b]{2}{*}{ Feedstock } & \multicolumn{4}{|c|}{ Reaction Conditions } & \multirow[b]{2}{*}{$\begin{array}{l}\text { Conversion/ } \\
\text { Biodiesel } \\
\text { Yield (\%) }\end{array}$} & \multirow[b]{2}{*}{$\begin{array}{c}\text { Time of } \\
\text { Reuse/Corresponding } \\
\text { Biodiesel Yield (\%) }\end{array}$} & \multirow[b]{2}{*}{ Ref. } \\
\hline & & $\begin{array}{c}\text { Catalyst } \\
\text { Loading (\%) }\end{array}$ & $\begin{array}{c}\text { Alcohol: } \\
\text { Fatty Acid } \\
\text { Molar Ratio }\end{array}$ & $\begin{array}{l}\text { Time } \\
(\mathrm{min})\end{array}$ & $\begin{array}{l}\text { Temp. } \\
\left({ }^{\circ} \mathrm{C}\right)\end{array}$ & & & \\
\hline $\begin{array}{c}\text { CaO-based catalyst } \\
\text { derived from } \\
\text { eggshell-snail } \\
\text { shell-wood ash } \\
\text { mixed }\end{array}$ & $\begin{array}{c}\text { Mixture of } \\
\text { Irvingia } \\
\text { gabonensis, } \\
\text { Pentaclethra } \\
\text { macrophylla, and } \\
\text { Elais } \\
\text { guineensis oil }\end{array}$ & 4.5 & $8: 1$ & 64.71 & 61.61 & 98 & $5 / 79$ & [82] \\
\hline $\begin{array}{l}\text { Ram bone } \\
\text { supported Cr } \\
\text { catalyst }\end{array}$ & $\begin{array}{l}\text { Used frying } \\
\text { mustard oil }\end{array}$ & 4 & $8: 1$ & 30 & 60 & 96.85 & $5 / 95.56$ & [83] \\
\hline $\begin{array}{l}\text { Lithium based } \\
\text { chicken bone } \\
\text { composite }\end{array}$ & Canola oil & 4 & 18:1 & 180 & 60 & 96.6 & $5 / 82$ & [84] \\
\hline $\begin{array}{l}\text { Lithium/zinc } \\
\text { supported on } \\
\text { chicken bone } \\
\text { catalyst }\end{array}$ & Waste canola oil & 4 & $18: 1$ & 210 & 60 & 98 & $7 />96$ & [85] \\
\hline $\begin{array}{l}\text { Goat bone-derived } \\
\text { nano-CaO }\end{array}$ & $\begin{array}{l}\text { Scenedesmus algal } \\
\text { oil }\end{array}$ & 2 & $11: 1$ & 180 & 60 & 92 & - & [86] \\
\hline $\begin{array}{l}\mathrm{KOH} \text { impregnated } \\
\mathrm{CaO} \text { derived from } \\
\text { goat bone }\end{array}$ & WCO & 6 & $9: 1$ & 300 & 65 & 84 & - & [87] \\
\hline $\begin{array}{l}\text { Chicken and fish } \\
\text { bone-derived } \mathrm{CaO}\end{array}$ & WCO & 1.98 & $10: 1$ & 92 & 65 & 89.5 & $5 /<50$ & [88] \\
\hline $\begin{array}{c}\text { Struthio camelus } \\
\text { bone-derived } \mathrm{CaO}\end{array}$ & WCO & 5 & $15: 1$ & 240 & 60 & 90.56 & $5 />80$ & [89] \\
\hline $\begin{array}{l}\text { Poly- glycidyl- } \\
\text { methacrylate } \\
\text { grafted flax fibers }\end{array}$ & Cottonseed oil & 2.5 & $33: 1$ & 120 & 60 & 88.6 & $3 / 72.5$ & [90] \\
\hline $\begin{array}{l}\text { Calcined cupuaçu } \\
\text { (Theobroma } \\
\text { grandiflorum) seeds }\end{array}$ & Soybean oil & $10 \%$ & $10: 1$ & 480 & 80 & 98.36 & $3 />20$ & [31] \\
\hline $\begin{array}{c}\mathrm{K}_{2} \mathrm{O}-\mathrm{KCl} \text { derived } \\
\text { from calcined } \\
\text { banana peel }\end{array}$ & Soybean oil & 1.5 & $15: 1$ & 60 & 65 & 95.1 & $4 / 75.5$ & [32] \\
\hline $\begin{array}{l}\text { Calcined husk of } \\
\text { Enterolobium } \\
\text { cyclocarpum pods }\end{array}$ & Oil blend & 2.96 & $11.44: 1$ & 5.88 & 65 & 98.77 & $4 / 74.68$ & [33] \\
\hline $\begin{array}{l}\text { Calcined kola nut } \\
\text { husk pod }\end{array}$ & $\begin{array}{c}\text { Hevea brasiliensis } \\
\text { seed oil }\end{array}$ & 3.5 & $6: 1$ & 75 & 65 & 96.97 & - & [34] \\
\hline $\begin{array}{l}\text { Calcined Brassica } \\
\text { nigra plant }\end{array}$ & Soybean oil & 7 & $12: 1$ & 25 & 65 & 98.79 & $3 />96$ & [35] \\
\hline $\begin{array}{c}\mathrm{ZrO}_{2} \text { supported on } \\
\text { bamboo leaf ash }\end{array}$ & Soybean oil & 12 & $15: 1$ & 30 & 50 & 92.75 & - & [36] \\
\hline $\begin{array}{l}\text { Calcined Sesamum } \\
\text { indicum ash }\end{array}$ & Sunflower oil & 7 & $12: 1$ & 40 & 65 & 98.9 & $3 / 94.2$ & [37] \\
\hline $\begin{array}{l}\text { Calcined Tectona } \\
\text { grandis leaves }\end{array}$ & WCO & 2.5 & $6: 1$ & 180 & RT & 100 & $4 />80$ & [38] \\
\hline $\begin{array}{c}\text { Calcined Ficus } \\
\text { carica leaves }\end{array}$ & WCO & 1 & $6: 1$ & 120 & 60 & 90.75 & - & [39] \\
\hline $\begin{array}{c}\text { Calcined ginger } \\
\text { (Zingiber officinale) } \\
\text { leaves activated by } \\
\mathrm{KOH}\end{array}$ & Sunflower oil & 1.6 & $6: 1$ & 90 & 60 & 93.83 & - & [40] \\
\hline
\end{tabular}


Table 1. Cont.

\begin{tabular}{|c|c|c|c|c|c|c|c|c|}
\hline \multirow[b]{2}{*}{ Catalyst } & \multirow[b]{2}{*}{ Feedstock } & \multicolumn{4}{|c|}{ Reaction Conditions } & \multirow[b]{2}{*}{$\begin{array}{l}\text { Conversion/ } \\
\text { Biodiesel } \\
\text { Yield (\%) }\end{array}$} & \multirow[b]{2}{*}{$\begin{array}{c}\text { Time of } \\
\text { Reuse/Corresponding } \\
\text { Biodiesel Yield (\%) }\end{array}$} & \multirow[b]{2}{*}{ Ref } \\
\hline & & $\begin{array}{c}\text { Catalyst } \\
\text { Loading (\%) }\end{array}$ & $\begin{array}{c}\text { Alcohol: } \\
\text { Fatty Acid } \\
\text { Molar Ratio }\end{array}$ & $\begin{array}{l}\text { Time } \\
(\min )\end{array}$ & $\begin{array}{l}\text { Temp. } \\
\left({ }^{\circ} \mathrm{C}\right)\end{array}$ & & & \\
\hline $\begin{array}{l}\text { Calcined Carica } \\
\text { papaya stem }\end{array}$ & WCO & 2 & $9: 1$ & 180 & 60 & 95.23 & $6 / 85.4$ & [41] \\
\hline $\begin{array}{l}\text { Calcined banana } \\
\text { peel }\end{array}$ & WCO & 2 & $6: 1$ & 180 & 60 & 100 & $3 / 66.66$ & {$[42]$} \\
\hline $\begin{array}{c}\mathrm{KOH} / \text { corncob- }^{-} \\
\text {derived activated } \\
\text { carbon }\end{array}$ & WCO & 1 & 18:1 & 60 & 45 & 97.8 & $2 / 35$ & [43] \\
\hline $\begin{array}{l}\text { Supermagnetic } \\
\text { catalyst derived } \\
\text { from rice husk } \\
\text { doped with } \mathrm{K}_{2} \mathrm{O} \\
\text { and } \mathrm{Fe}\end{array}$ & WCO & 4 & $12: 1$ & 240 & 75 & 98.6 & $5 />80$ & {$[44]$} \\
\hline $\begin{array}{l}\mathrm{CaO} / \text { zeolite-based } \\
\text { catalyst derived } \\
\text { from chicken } \\
\text { eggshell and coal } \\
\text { fly ash }\end{array}$ & Sunflower oil & 6 & $6: 1$ & 30 & 60 & 97.8 & - & [54] \\
\hline Orange peel ash & Soybean oil & 7 & $6: 1$ & 420 & RT & 98 & $5 / 85$ & [91] \\
\hline $\begin{array}{l}\text { Rice husk biochar } \\
\text { supported } \mathrm{CaO}\end{array}$ & Palm oil & 8 & $9: 1$ & 180 & 65 & 93.4 & $10 / 85$ & [92] \\
\hline $\begin{array}{l}\text { Silica impregnated } \\
\mathrm{CaO} \text { derived from } \\
\text { eggshell }\end{array}$ & $\begin{array}{l}\text { Virgin cooking } \\
\text { palm oil }\end{array}$ & 3 & $20: 1$ & 120 & 60 & 87.5 & $6 />80$ & [93] \\
\hline Sugarcane leaf ash & $\begin{array}{l}\text { Calophyllum } \\
\text { inophyllum oil }\end{array}$ & 5 & 19:1 & 180 & 64 & 97 & $10 / 74$ & [94] \\
\hline $\begin{array}{c}\mathrm{SiO}_{2} \text {-rich } \\
\text { sugarcane bagasse } \\
\text { ash }\end{array}$ & Palm oil & 6 & $20: 1$ & 180 & 65 & 93.8 & $5 / 70.3$ & [95] \\
\hline $\begin{array}{l}\text { Calcined barnacles } \\
\text { shell }\end{array}$ & $\begin{array}{l}\text { Aglaia korthalsii } \\
\text { seed oil }\end{array}$ & 4.7 & $12.2: 1$ & 180 & 65 & 97.12 & $4 / 95.83$ & [96] \\
\hline $\begin{array}{l}\text { Calcined banana } \\
\text { peduncle }\end{array}$ & $\begin{array}{l}\text { Ceiba pentandra } \\
\text { oil }\end{array}$ & 1.978 & $9.2: 1$ & 60 & 65 & 98.69 & - & [97] \\
\hline $\begin{array}{l}\text { Silica-supported } \\
\mathrm{CaO} \text { derived from } \\
\text { goat bone }\end{array}$ & WCO & 6 & $15: 1$ & 120 & 60 & 94 & $7 / 40$ & [98] \\
\hline $\begin{array}{l}\text { Calcined quail } \\
\text { beaks }\end{array}$ & Rapeseed oil & 7 & $12: 1$ & 240 & 65 & 96.7 & $6 />90$ & [99] \\
\hline $\begin{array}{c}\text { Calcined walnut } \\
\text { shell }\end{array}$ & Sunflower oil & 5 & $12: 1$ & 10 & 60 & 98 & $4 />95$ & [100] \\
\hline
\end{tabular}

RT: room temperature.

\section{Bio-Derived Acid Catalysts for Biodiesel Production}

Alkali-catalyzed transesterification is efficient for producing biodiesel from refined oils (containing a low level of free fatty acids (FFA)). However, the biodiesel yield is significantly reduced when the oil contains a high level of FFA $(>1 \%, w / w)$ because alkali catalysts cannot convert FFA into biodiesel and the liquid alkali catalysts can react with FFA to form soap [2]. Therefore, acid-catalyzed esterification/transesterification is commonly proposed to produce biodiesel from high FFA-containing oils. Acid catalysts simultaneously catalyze the esterification of FAA and transesterification of oil (triglyceride) into biodiesel; therefore, they are insensitive to the quality of the raw material. In addition, the use of the acid catalysts for biodiesel production prevents the saponification reaction, which is commonly found in the homogenous alkali-catalyzed transesterification reaction. Homogenous acid catalysts (such as $\mathrm{HCl}, \mathrm{H}_{2} \mathrm{SO}_{4}, \mathrm{H}_{3} \mathrm{PO}_{4}$ ) are widespread in biodiesel production because 
they efficiently convert FFA and triglyceride into biodiesel $[9,10]$. However, there are lots of associated problems in the downstream process, which is costly and requires complicated steps for product purification and separation of the catalyst. In addition, the use of these homogenous acid catalysts causes corrosive damage to the equipment and negatively affects the environment. These liquid catalysts are also difficult recover and reuse. To address these obstacles, heterogenous/solid acid catalysts have been increasingly considered as promising alternative catalysts to facilitate a cleaner, safer, simpler, and cheaper process for biodiesel production [101,102].

In recent years, biomass-derived acid catalysts have gained much interest in biodiesel production due to their ecofriendly properties, potential reusability, and the availability and low cost of materials used for catalyst synthesis. Recently, several forms of heterogeneous acid carbon-based catalysts have been developed for biodiesel production from high-FFA oils. The carbonization followed by sulfonation method is commonly used to synthesize various solid acid catalysts such as sulfonated carbon from corn cobs [103], sulfonated starch [104], sulfonated carbon from vegetable oil asphalt [105], sulfonated carbon from cacao shell [106], sulfonated rice husk [107], sulfonated bamboo [108], sulfonated sugarcane bagasse [109], sulfonated biochar derived from cassava peel [110], and sulfonated biochar derived from sugarcane bagasse, corncob, coconut shell, and peanut shell [111]. Different materials result in different catalytic activities of the synthesized catalysts. The catalysts prepared from these materials demonstrated good catalytic efficiency towards esterification of high-FFA oils, with FFA conversions ranging from $71 \%$ to $98 \%[109,110]$. Among the materials used, waste shells, such as cacao shell [106], wing shell [112], and coconut shell [113], show promise for the synthesis of solid acid catalysts. More acid catalysts used for biodiesel production are shown in Table 2. In comparison with alkali catalysts, the bio-based acid-catalyzed reaction commonly requires a longer reaction time and higher temperature for biodiesel production. Therefore, the acid-catalyzed reaction is only suggested for producing biodiesel from feedstock containing a high level of FFA.

Table 2. Several bio-derived acid catalysts for biodiesel production.

\begin{tabular}{|c|c|c|c|c|c|c|c|c|}
\hline \multirow[b]{2}{*}{ Catalyst } & \multirow[b]{2}{*}{ Feedstock } & \multicolumn{4}{|c|}{ Reaction Conditions } & \multirow[b]{2}{*}{$\begin{array}{l}\text { Conversion/ } \\
\text { Biodiesel } \\
\text { Yield (\%) }\end{array}$} & \multirow[b]{2}{*}{$\begin{array}{c}\text { Time of } \\
\text { Reuse/Corresponding } \\
\text { Biodiesel Yield (\%) }\end{array}$} & \multirow[b]{2}{*}{ Ref. } \\
\hline & & $\begin{array}{c}\text { Catalyst } \\
\text { Loading (\%) }\end{array}$ & $\begin{array}{c}\text { Alcohol: } \\
\text { Fatty Acid } \\
\text { Molar Ratio }\end{array}$ & $\begin{array}{l}\text { Time } \\
\text { (min) }\end{array}$ & $\begin{array}{l}\text { Temp. } \\
\left({ }^{\circ} \mathrm{C}\right)\end{array}$ & & & \\
\hline $\begin{array}{l}\text { Sulfonated- } \\
\text { carbonized } \\
\text { bamboo }\end{array}$ & Oleic acid & 5 & $8: 1$ & 60 & 65 & 97.31 & $5 /<40$ & [108] \\
\hline $\begin{array}{l}\text { Sulfated angel } \\
\text { wing shells }\end{array}$ & $\begin{array}{l}\text { Palm fatty acid } \\
\text { distillate }\end{array}$ & 2 & $6: 1$ & 15 & 290 & 98 & $7 />80$ & [112] \\
\hline $\begin{array}{l}\text { Sulfonated- } \\
\text { carbonized coconut } \\
\text { shell }\end{array}$ & Palm oil & 6 & $30: 1$ & 360 & 60 & 88.15 & - & [113] \\
\hline $\begin{array}{c}\text { Sulfated- } \\
\text { carbonized Jatropha } \\
\text { curcas seed }\end{array}$ & Jaropha curcas oil & 7.5 & $12: 1$ & 60 & 60 & 99.13 & $4 / 81.03$ & [114] \\
\hline $\begin{array}{l}\text { Carbonaceous } \\
\text { solid acid magnetic } \\
\text { catalyst from } \\
\text { empty fruit bunch }\end{array}$ & $\begin{array}{l}\text { Palm fatty acid } \\
\text { distillate }\end{array}$ & 4 & $16: 1$ & 180 & 100 & 98.6 & $6 / 79$ & [115] \\
\hline $\begin{array}{l}\text { Sulfonated cow } \\
\text { dung-derived } \\
\text { carbon-based } \\
\text { catalyst }\end{array}$ & $\begin{array}{l}\text { Palm fatty acid } \\
\text { distillate }\end{array}$ & 4 & $18: 1$ & 60 & 90 & 96.5 & $7 / 75$ & [116] \\
\hline $\begin{array}{c}\text { CaO-based } \\
\text { calcined angel } \\
\text { wing shell sulfated } \\
\text { catalyst }\end{array}$ & $\begin{array}{l}\text { Palm fatty acid } \\
\text { distillate }\end{array}$ & 5 & $15: 1$ & 180 & 80 & 98 & $4 />40$ & [117] \\
\hline
\end{tabular}


Table 2. Cont.

\begin{tabular}{|c|c|c|c|c|c|c|c|c|}
\hline \multirow[b]{2}{*}{ Catalyst } & \multirow[b]{2}{*}{ Feedstock } & \multicolumn{4}{|c|}{ Reaction Conditions } & \multirow[b]{2}{*}{$\begin{array}{l}\text { Conversion/ } \\
\text { Biodiesel } \\
\text { Yield (\%) }\end{array}$} & \multirow[b]{2}{*}{$\begin{array}{c}\text { Time of } \\
\text { Reuse/Corresponding } \\
\text { Biodiesel Yield (\%) }\end{array}$} & \multirow[b]{2}{*}{ Ref. } \\
\hline & & $\begin{array}{c}\text { Catalyst } \\
\text { Loading (\%) }\end{array}$ & $\begin{array}{c}\text { Alcohol: } \\
\text { Fatty Acid } \\
\text { Molar Ratio }\end{array}$ & $\begin{array}{l}\text { Time } \\
(\min )\end{array}$ & $\begin{array}{l}\text { Temp. } \\
\left({ }^{\circ} \mathrm{C}\right)\end{array}$ & & & \\
\hline $\begin{array}{l}\text { Sulfonated- } \\
\text { carbonated coconut } \\
\text { meal residue }\end{array}$ & $\begin{array}{l}\text { Waste palm } \\
\text { cooking oil }\end{array}$ & 5 & 12:1 & 180 & 150 & 95.5 & $4 / 82$ & [118] \\
\hline $\begin{array}{l}\text { Sulfonated carbon } \\
\text { derived from } \\
\text { coconut meal } \\
\text { residue }\end{array}$ & Waste cooking oil & 6 & $9: 1$ & 300 & 65 & 96 & - & [119] \\
\hline $\begin{array}{l}\text { Sulfated Ce } \\
\text { supported } \\
\text { activated carbon } \\
\text { derived from } \\
\text { coconut shell }\end{array}$ & Chicken fat oil & 3 & $12: 1$ & 60 & 90 & 93 & $5 / 90$ & [120] \\
\hline $\begin{array}{l}\text { Sulfonated and } \\
\text { magnetic catalyst } \\
\text { derived from palm } \\
\text { kernel shell }\end{array}$ & Waste cooking oil & 3.66 & 13:1 & 102 & 65 & 90.2 & $4 / 73.63$ & [121] \\
\hline $\begin{array}{c}\text { Sulfonated } \\
\text { carbon-based } \\
\text { catalysts from } \\
\text { murumuru kernel } \\
\text { shell }\end{array}$ & Oleic acid & 5 & $10: 1$ & 90 & 90 & 97.2 & $4 / 66.3$ & [122] \\
\hline $\begin{array}{c}\text { Sulfonated } \\
\text { carbon-based } \\
\text { catalyst from } \\
\text { Murumuru kernel } \\
\text { shell }\end{array}$ & Jupati oil & 6 & $30: 1$ & 240 & 135 & 91.8 & $4 />80$ & [123] \\
\hline $\begin{array}{l}\text { Sulfonated biochar } \\
\text { derived from } \\
\text { sawdust }\end{array}$ & $\begin{array}{c}\text { Pongamia pinnatta } \\
\text { oil }\end{array}$ & 2 & $9: 1$ & 120 & 85 & 95.6 & $4 / 85.7$ & [124] \\
\hline $\begin{array}{c}\text { Sulfonated- } \\
\text { carbonized } \\
\text { Zanthoxylum } \\
\text { bungeanum seed }\end{array}$ & $\begin{array}{c}\text { Zanthoxylum } \\
\text { bungeanum seed } \\
\text { oil }\end{array}$ & 8 & $30: 1$ & 240 & 140 & 95.6 & $5 / 57 / 9$ & [125] \\
\hline $\begin{array}{c}\text { Sulfonated- } \\
\text { calcined kenaf seed } \\
\text { cake }\end{array}$ & $\begin{array}{l}\text { Palm fatty acid } \\
\text { distillate }\end{array}$ & 2 & 10:1 & 90 & 65 & 97.9 & $5 />90$ & [126] \\
\hline $\begin{array}{c}\text { Palm } \\
\text { biochar-based } \\
\text { sulfated zirconium }\end{array}$ & $\begin{array}{l}\text { Palm fatty acid } \\
\text { distillate }\end{array}$ & 3 & $15: 1$ & 180 & 75 & 94.3 & $5 / 80.2$ & [127] \\
\hline $\begin{array}{c}\text { Sulfonated } \\
\text { activated carbon } \\
\text { derived from Monk } \\
\text { fruit seed (Siraitia } \\
\text { grosvenorii) }\end{array}$ & & 4 & & 360 & 120 & 98.5 & $4 / 84.4$ & [128] \\
\hline $\begin{array}{l}\text { Sulfonated- } \\
\text { derived tea } \\
\text { waste }\end{array}$ & $\begin{array}{l}\text { Palm fatty acid } \\
\text { distillate }\end{array}$ & 4 & $9: 1$ & 90 & 65 & 97 & $5>80$ & [129] \\
\hline $\begin{array}{c}\text { Sulfonated- } \\
\text { carbonized Hura } \\
\text { crepitans seed pod }\end{array}$ & $\begin{array}{c}\text { High-FFA } \\
\text { vegetable oil }\end{array}$ & 10 & $9: 1$ & 60 & 90 & 94.81 & 4/93.37 & [130] \\
\hline $\begin{array}{l}\text { Sulfonated- } \\
\text { carbonized cotton } \\
\text { stalk }\end{array}$ & $\begin{array}{l}\text { Madhuca indica } \\
\text { oil }\end{array}$ & 5 & 18:1 & 300 & 60 & 89.2 & $7 / 83.4$ & [131] \\
\hline
\end{tabular}


Table 2. Cont

\begin{tabular}{|c|c|c|c|c|c|c|c|c|}
\hline \multirow[b]{2}{*}{ Catalyst } & \multirow[b]{2}{*}{ Feedstock } & \multicolumn{4}{|c|}{ Reaction Conditions } & \multirow[b]{2}{*}{$\begin{array}{l}\text { Conversion/ } \\
\text { Biodiesel } \\
\text { Yield (\%) }\end{array}$} & \multirow[b]{2}{*}{$\begin{array}{c}\text { Time of } \\
\text { Reuse/Corresponding } \\
\text { Biodiesel Yield (\%) }\end{array}$} & \multirow[b]{2}{*}{ Ref. } \\
\hline & & $\begin{array}{c}\text { Catalyst } \\
\text { Loading (\%) }\end{array}$ & $\begin{array}{c}\text { Alcohol: } \\
\text { Fatty Acid } \\
\text { Molar Ratio }\end{array}$ & $\begin{array}{l}\text { Time } \\
(\min )\end{array}$ & $\begin{array}{l}\text { Temp. } \\
\left({ }^{\circ} \mathrm{C}\right)\end{array}$ & & & \\
\hline $\begin{array}{c}\text { Sulfonated } \\
\text { activated carbon } \\
\text { derived from } \\
\text { Mesua ferrea shell }\end{array}$ & Mesua ferrea oil & 10 & $6: 1$ & 120 & 55 & 95.57 & - & [132] \\
\hline $\begin{array}{l}\text { Sulfonated biochar } \\
\text { derived from palm } \\
\text { empty fruit bunch }\end{array}$ & $\begin{array}{l}\text { Palm fatty acid } \\
\text { distillate }\end{array}$ & 20 & $30: 1$ & 420 & 110 & 98.1 & - & [133] \\
\hline $\begin{array}{l}\text { Sulfonated carbon } \\
\text { derived from } \\
\text { corncob residue }\end{array}$ & $\begin{array}{l}\text { Palm fatty acid } \\
\text { distillate }\end{array}$ & 3 & $15: 1$ & 120 & 70 & 85 & $5 / 60$ & [134] \\
\hline $\begin{array}{l}\text { Sulfonated carbon } \\
\text { derived from } \\
\text { coconut meal } \\
\text { residue }\end{array}$ & Waste palm oil & 5 & $12: 1$ & 720 & $65-70$ & 92.7 & $4 />80$ & [135] \\
\hline $\begin{array}{l}\text { Sulfonated- } \\
\text { carbonized spent } \\
\text { coffee grounds }\end{array}$ & Oleic acid & 10 & $10: 1$ & 420 & 80 & 91.2 & $4 / 26.41$ & [136] \\
\hline $\begin{array}{l}\text { Sulfonated pine } \\
\text { needle-derived } \\
\text { carbon }\end{array}$ & Levulinic acid & 5 & $5: 1$ & 480 & 80 & 96.1 & $4 />60$ & [137] \\
\hline $\begin{array}{c}\text { Sulfonated rice } \\
\text { husk }\end{array}$ & Oleic acid & 5 & $5: 1$ & 20 & 28 & 99.8 & $3 / 70$ & [138] \\
\hline $\begin{array}{l}\text { Sulfonated rubber } \\
\text { de-oiled cake }\end{array}$ & Waste cooking oil & 8.18 & $12.8: 1$ & 60 & 63 & 91.2 & $3 / 80$ & [139] \\
\hline $\begin{array}{c}\text { Magnetic } \\
\text { carbonaceous acid } \\
\text { derived from } \\
\text { Jatropha hulls }\end{array}$ & Jatropha crude oil & 7.5 & $18: 1$ & 450 & 180 & 95.9 & $5 / 94.3$ & [140] \\
\hline $\begin{array}{l}\text { Sulfonated carbon } \\
\text { derived from } \\
\text { potato peel }\end{array}$ & Oleic acid & 5 & $12: 1$ & 150 & 80 & 97.2 & $5 / 68$ & [141] \\
\hline $\begin{array}{l}\text { Sulfonated waste } \\
\text { yeast residue }\end{array}$ & Waste cooking oil & 1 & 10:1 & 360 & 60 & 96.2 & $6 /<80$ & [142] \\
\hline $\begin{array}{c}\text { Sulfonated- } \\
\text { carbonized cacao } \\
\text { shell } \\
\end{array}$ & Oleic acid & 5 & $7: 1$ & 1440 & 45 & 94 & $4 /<50$ & [143] \\
\hline $\begin{array}{l}\text { Sulfonated coconut } \\
\text { coir husk }\end{array}$ & Waste palm oil & 10 & $12: 1$ & 180 & 130 & 89.8 & $4 /<80$ & [144] \\
\hline $\begin{array}{c}\text { Sulfonated } \\
\text { lignin-derived } \\
\text { from olive cake }\end{array}$ & $\begin{array}{l}\text { Waste vegetable } \\
\text { oil }\end{array}$ & 10 & $35: 1$ & 360 & 65 & 57 & $10 / 75$ & [145] \\
\hline $\begin{array}{l}\text { Sulfonated soaked } \\
\text { palm seed cake } \\
\text { derived catalyst }\end{array}$ & $\begin{array}{l}\text { Palm fatty acid } \\
\text { distillate (PFAD) }\end{array}$ & 2.5 & $9: 1$ & 120 & 60 & 97.8 & - & [146] \\
\hline $\begin{array}{l}\text { Sulfonated- } \\
\text { calcined corncobs } \\
\text { and calcined } \\
\text { poultry }\end{array}$ & Neem seed oil & 2.58 & $14.76: 1$ & 72.65 & 61.90 & 92.89 & $4 / 76$ & [147] \\
\hline $\begin{array}{c}\text { Sulfonated } \\
\text { brewer's spent } \\
\text { yeast }\end{array}$ & $\begin{array}{l}\text { Palm fatty acid } \\
\text { distillate }\end{array}$ & 8 & $21: 1$ & 180 & 65 & 87.8 & - & [148] \\
\hline
\end{tabular}

\section{Enzyme}

With an increasing demand for environmental protection, green processes have been rapidly developed for chemical production. Consequently, various ecofriendly processes 
have been proposed for producing biodiesel to reduce the adverse environmental effects $[5,149]$. Particularly, the enzyme-catalyzed reaction is one of the most promising processes for biodiesel production due to the ecofriendly and reusable nature of the enzyme. Notably, the enzymatic process proceeds at mild reaction temperature and pressure, thus lowering the energy consumption [150]. For this approach, biodiesel can be produced via lipase-catalyzed transesterification or lipase-catalyzed hydroesterification processes (hydrolysis of oils into FFA followed by esterification of the produced FFA with short-chain alcohols). The lipase catalyzes the esterification and transesterification simultaneously; therefore, the enzymatic process is insensitive to high-FFA oil [150]. Because of such benefits, enzymatic processes have been widely developed for biodiesel production from various feedstocks [150].

The efficiency of the enzymatic process mainly depends on the activity of lipases. Therefore, a great effort has been made to use lipase from different sources (microorganisms, plants, animals) for biodiesel production [151,152] (Table 3). The most common source of the lipase is microorganisms such as Candida antarctica [153,154], Thermomyces lanuginosu [155,156], Rhizomucor miehei [157,158], Pseudomonas cepacia [159,160], Candida rugosa [161,162], Aspergillus oryzae [163], Burkholderia cepacia [164,165], Adansonia grandidieri [166], Rhizopus oryzae [167], Pseudomonas fluorescens [168], Lactobacillus plantarum [169], and Aspergillus terreus [170]. Lipases from microorganisms are mainly used for biodiesel production due to the availability of sources and rapid growth rate of microorganisms for enzyme production [171]. Lipase activity depends not only on the source of the enzyme, but also the type of enzyme used (immobilized form or liquid form) [171]. Immobilizing lipase on the support material can enhance the stability of the enzyme, making the enzyme less susceptible to the $\mathrm{pH}$, temperature, and impurities of reactants [171]. Notably, the supports and/or immobilization protocols can greatly modulate the specific activity of lipase, affecting biodiesel yield. Tacias-Pascacio et al. [172] immobilized different lipases on different supports and used them for biodiesel production. They found that the specific activity of lipases and biodiesel yield greatly depended on the support, solvent used, and media [172]. In addition, the immobilized enzyme is easy to reuse. Consequently, lipase immobilized on various supporting materials has been studied for biodiesel production. Recently, Iuliano et al. [173] reported that lipase from C. rugosa was physically attached to $\mathrm{Mg}$ modified $\mathrm{Fe}_{2} \mathrm{O}_{4}$ nanoparticles and used to turn brewers' spent grains into biodiesel. After $48 \mathrm{~h}$ at $45{ }^{\circ} \mathrm{C}$, a remarkable yield of $98 \%$ was achieved using a 1:4 oil/methanol molar ratio. In addition, lipases were immobilized on other materials such as graphene oxide [174], polyhydroxyalkanoate [175], alginate-polyvinyl alcohol (PVA) [167], polydopamine coated iron oxide $\left(\mathrm{Fe}_{3} \mathrm{O}_{4}\right.$ PDA_lipase) [170], modified polyporous magnetic cellulose support [153], $\mathrm{Co}^{2+}$-chelated magnetic nanoparticles [168], core-shell structured $\mathrm{Fe}_{3} \mathrm{O}_{4} @ \mathrm{MIL}-100(\mathrm{Fe})$ composites [162], $\mathrm{Fe}_{3} \mathrm{O}_{4} / \mathrm{Au}$ nanoparticles [176], waste-derived activated carbon support [177], genipin cross-linked chitosan [178], and other materials [160]. Several immobilized lipases have been commercialized and used for biodiesel production such as Novozym ${ }^{\circledR} 435$ (lipase B from C. antarctica) [179-181] and Lipozyme TL IM (lipase from T. lanuginosus) [182]. Nevertheless, the immobilized lipase-catalyzed reaction rate is relatively low due to the mass transfer limitation between the substrate and enzyme [183]. Notably, the immobilized lipases are expensive, thus limiting their industrial applications. 
Table 3. Several lipases used for biodiesel production.

\begin{tabular}{|c|c|c|c|c|c|c|c|c|}
\hline \multirow[b]{2}{*}{ Catalyst } & \multirow[b]{2}{*}{ Feedstock } & \multicolumn{4}{|c|}{ Reaction Conditions } & \multirow[b]{2}{*}{$\begin{array}{l}\text { Conversion/ } \\
\text { Biodiesel } \\
\text { Yield (\%) }\end{array}$} & \multirow[b]{2}{*}{$\begin{array}{c}\text { Time of } \\
\text { Reuse/Corresponding } \\
\text { Biodiesel Yield }\end{array}$} & \multirow[b]{2}{*}{ Ref. } \\
\hline & & $\begin{array}{c}\text { Catalyst } \\
\text { Loading (\%) }\end{array}$ & $\begin{array}{c}\text { Alcohol: } \\
\text { Fatty Acid } \\
\text { Molar Ratio }\end{array}$ & $\begin{array}{l}\text { Time } \\
\text { (h) }\end{array}$ & $\begin{array}{l}\text { Temp. } \\
\left({ }^{\circ} \mathrm{C}\right)\end{array}$ & & & \\
\hline $\begin{array}{l}\text { C. antarctica lipase } \\
\text { B (CALB) } \\
\text { immobilized on } \\
\text { modified } \\
\text { polyporous } \\
\text { magnetic cellulose } \\
\text { beads }\end{array}$ & $\begin{array}{l}\text { Yellow horn seed } \\
\text { oil }\end{array}$ & 15 & $1.6: 1$ & 2 & 60 & 92.3 & $5 / 85$ & [153] \\
\hline $\begin{array}{c}\text { C. antarctica lipase } \\
\text { A (CALA) }\end{array}$ & Palm oil & 5.5 & $7: 1$ & 22 & 30 & 94.6 & - & [154] \\
\hline $\begin{array}{l}\text { Novozym }^{\circledR} 435 \\
\text { (CALB } \\
\text { immobilized on } \\
\text { macroporous } \\
\text { acrylic resin) }\end{array}$ & $\begin{array}{l}\text { Residual babassu } \\
\text { oil }\end{array}$ & $0.14 \mathrm{~g}$ & $18: 1$ & 4 & 48 & 96.8 & $10 / 90.96$ & [179] \\
\hline Novozyme $^{\circledR} 435$ & $\begin{array}{l}\text { Castor oil fatty } \\
\text { acid }\end{array}$ & 10 & $3: 1$ & 5 & 60 & 88.64 & - & [180] \\
\hline Novozyme $^{\circledR} 435$ & Spirogyra oil & 1 & $4.5: 1$ & 42.5 & 35 & 93.2 & - & [184] \\
\hline Novozym $^{\circledR} 435$ & $\begin{array}{l}\text { Black soldier fly } \\
\text { larvae oil }\end{array}$ & 17.58 & $14.64: 1$ & 12 & 39.5 & 96.97 & $20 />95$ & [185] \\
\hline CALA & Soybean oil & 5 & $7: 1$ & 26 & 38 & 92.4 & - & [186] \\
\hline $\begin{array}{c}\text { CALB immobilized } \\
\text { on methacrylic } \\
\text { resin }\end{array}$ & Waste animal fat & 14 & $10: 1$ & 6 & 40 & 87 & - & [187] \\
\hline $\begin{array}{l}\text { CALB immobilized } \\
\text { on magnetic } \\
\text { nanoparticles }\end{array}$ & $\begin{array}{l}\text { Palm fatty acid } \\
\text { distillate }\end{array}$ & 8 & $1.6: 1$ & 10 & 50 & 82.74 & $5 / 80.19$ & [188] \\
\hline $\begin{array}{c}\text { CALB immobilized } \\
\text { magnetic } \\
\text { nanoparticles }\end{array}$ & Microalgal oil & 1 & $10: 1$ & 3 & 30 & 91.4 & $4 / 90$ & [189] \\
\hline $\begin{array}{c}67 \% \text { CALB }+33 \% \\
\text { lipase from } R . \\
\text { miehei }\end{array}$ & $\begin{array}{l}\text { Residual chicken } \\
\text { oil }\end{array}$ & 15 & $5: 1$ & 3 & 30 & 89.95 & - & [190] \\
\hline CALB & Soybean oil & 3 & $3: 1$ & 15 & 40 & 64.7 & - & [191] \\
\hline $\begin{array}{c}\text { CALB immobilized } \\
\text { on silica } \\
\text { nanoflowers }\end{array}$ & Waste oil & $33.24 \mathrm{mg}$ & 2.63:1 & 8.11 & 45.97 & 98.5 & $15 / 76.68$ & [192] \\
\hline $\begin{array}{c}\text { CALB and } \\
\text { Rhizomucor miehei } \\
\text { lipase } \\
\text { co-immobilized on } \\
\text { epoxy } \\
\text { functionalized } \\
\text { silica gel }\end{array}$ & Palm oil & $4.9 \mathrm{U} / \mathrm{mg}$ & $5.9: 1$ & 33.5 & 35.6 & 78.3 & - & [193] \\
\hline $\begin{array}{l}\text { Lipozyme TL100L } \\
\text { (T. lanuginosu } \\
\text { lipase) }\end{array}$ & $\begin{array}{l}\text { Waste phoenix } \\
\text { seed }\end{array}$ & 9.7 & $4.3: 1$ & 6.9 & 31 & 93.8 & - & [155] \\
\hline $\begin{array}{l}\text { Lipozyme TL IM } \\
\text { (immobilized T. } \\
\text { lanuginosus lipase) }\end{array}$ & Rapeseed oil & 5 & $5: 1$ & 5 & 25 & 98.76 & - & [156] \\
\hline Lipozyme TL IM & $\begin{array}{l}\text { Ankistrodesmus } \\
\text { sp. oil }\end{array}$ & 9.6 & $8: 1$ & 12 & 42 & 97.69 & & [194] \\
\hline
\end{tabular}


Table 3. Cont.

\begin{tabular}{|c|c|c|c|c|c|c|c|c|}
\hline \multirow[b]{2}{*}{ Catalyst } & \multirow[b]{2}{*}{ Feedstock } & \multicolumn{4}{|c|}{ Reaction Conditions } & \multirow[b]{2}{*}{$\begin{array}{l}\text { Conversion/ } \\
\text { Biodiesel } \\
\text { Yield (\%) }\end{array}$} & \multirow[b]{2}{*}{$\begin{array}{c}\text { Time of } \\
\text { Reuse/Corresponding } \\
\text { Biodiesel Yield }\end{array}$} & \multirow[b]{2}{*}{ Ref. } \\
\hline & & $\begin{array}{c}\text { Catalyst } \\
\text { Loading (\%) }\end{array}$ & $\begin{array}{c}\text { Alcohol: } \\
\text { Fatty Acid } \\
\text { Molar Ratio }\end{array}$ & $\begin{array}{c}\text { Time } \\
\text { (h) }\end{array}$ & $\begin{array}{l}\text { Temp. } \\
\left({ }^{\circ} \mathrm{C}\right)\end{array}$ & & & \\
\hline $\begin{array}{c}\text { T. lanugionous } \\
\text { immobilized on } \\
\mathrm{Fe}_{3} \mathrm{O}_{4} \\
\text { nanoparticles }\end{array}$ & Soybean oil & 9 & $4: 1$ & 28 & 41 & 82.2 & $10 / 71.23$ & [182] \\
\hline $\begin{array}{l}\text { Lipase NS } 40116 \\
\text { (liquid lipase } \\
\text { formulation } \\
\text { derived from } T . \\
\text { lanuginosus) } \\
\end{array}$ & $\begin{array}{l}\text { Residual chicken } \\
\text { oil }\end{array}$ & 0.3 & $4: 1$ & 36 & 35 & 93.16 & - & [195] \\
\hline Lipozyme TL IM & Rapeseed oil & 5 & $9: 1$ & 7 & 30 & 99.89 & - & [196] \\
\hline Lipozyme TL100L & $\begin{array}{l}\text { Phoenix tree seed } \\
\text { oil }\end{array}$ & 10 & $5: 1$ & 6.98 & 30 & 98.8 & - & [197] \\
\hline Lipase NS 40116 & Soybean oil & 0.7 & $6.3: 1$ & 8 & 35 & 97.1 & - & [198] \\
\hline Lipase NS 40116 & Soybean oil & 0.5 & $4.5: 1$ & 12 & 35 & 94.3 & $5 / 90$ & [199] \\
\hline $\begin{array}{c}\text { T. lanuginosus } \\
\text { lipase immobilized } \\
\text { on Immobead } 150\end{array}$ & None-edible oils & 3.55 & $7.64: 1$ & 2 & 36 & 90 & - & [200] \\
\hline $\begin{array}{l}\text { T. lanuginosus } \\
\text { lipase immobilized } \\
\text { on } \mathrm{Fe}_{3} \mathrm{O}_{4} / \mathrm{Au} \\
\text { nanoparticles }\end{array}$ & Tomato seeds oil & 20 & $6: 1$ & 24 & 45 & 98.5 & $5 / 68.95$ & [176] \\
\hline $\begin{array}{l}\text { Liquid formulation } \\
\text { of } T \text {. lanuginosus } \\
\text { lipase }\end{array}$ & $\begin{array}{l}\text { Palm oil mill } \\
\text { effluent }\end{array}$ & $2100 \mathrm{U}$ & $4: 1$ & 24 & 40 & 97.43 & - & [201] \\
\hline $\begin{array}{l}\text { Eversa Transform } \\
\text { lipase (liquid lipase } \\
\text { from T. lanuginosus) }\end{array}$ & Oleic acid & 11.98 & $3.44: 1$ & 2.5 & 35.25 & 96.73 & $5 /<30$ & [183] \\
\hline R. miehei lipases & Oleic acid & 20 & $2: 1$ & 4 & 40 & 85 & $4 / 74$ & [157] \\
\hline $\begin{array}{c}\text { R. miehei lipase } \\
\text { immobilized on } \\
\text { magnetic } \\
\text { nanoparticles }\end{array}$ & Babassu oil & 5 & $1: 1$ & 6 & 40 & 81.7 & - & [158] \\
\hline $\begin{array}{l}\text { C. rugosa } \\
\text { immobilized on } \\
\text { polyhydroxybu- } \\
\text { tyrate + R. miehei } \\
\text { immobilized on } \\
\text { polyhydroxybu- } \\
\text { tyrate }\end{array}$ & WCO & 1 & $6: 1$ & 24 & 45 & 96.5 & $10 / 28.95$ & [175] \\
\hline $\begin{array}{c}\text { Mixture of } \\
\text { polyhydroxybutyrate- } \\
\text { immobilized C. } \\
\text { rugosa and } R \text {. miehei } \\
\text { lipases }\end{array}$ & $\begin{array}{l}\text { Mixed chicken } \\
\text { waste oil }\end{array}$ & 2.5 & $6: 1$ & 12 & 40 & 97.1 & $15 / 10$ & [202] \\
\hline $\begin{array}{c}\text { P. cepacia lipase } \\
\text { immobilized on } \\
\text { bio-support beads. }\end{array}$ & $\begin{array}{c}\text { Hybrid } \\
\text { non-edible oil }\end{array}$ & 10 & $6: 1$ & 24 & 50 & 78 & $12 / 19.5$ & [159] \\
\hline $\begin{array}{l}\text { P. cepacia lipase } \\
\text { immobilized on } \\
\text { bio-support beads }\end{array}$ & $\begin{array}{l}\text { Non-edible } \\
\text { hybrid oil }\end{array}$ & 9.46 & $5.93: 1$ & 24.32 & 49.7 & 84.58 & $10 />70$ & [160] \\
\hline $\begin{array}{c}\text { P. cepacia lipase } \\
\text { immobilized on } \\
\text { hybrid } \\
\text { PVA/AlgNa }\end{array}$ & Crude castor oil & 10 & $6: 1$ & 24 & 50 & 78 & $6 / 70$ & [203] \\
\hline
\end{tabular}


Table 3. Cont

\begin{tabular}{|c|c|c|c|c|c|c|c|c|}
\hline \multirow[b]{2}{*}{ Catalyst } & \multirow[b]{2}{*}{ Feedstock } & \multicolumn{4}{|c|}{ Reaction Conditions } & \multirow[b]{2}{*}{$\begin{array}{l}\text { Conversion/ } \\
\text { Biodiesel } \\
\text { Yield (\%) }\end{array}$} & \multirow[b]{2}{*}{$\begin{array}{c}\text { Time of } \\
\text { Reuse/Corresponding } \\
\text { Biodiesel Yield }\end{array}$} & \multirow[b]{2}{*}{ Ref. } \\
\hline & & $\begin{array}{c}\text { Catalyst } \\
\text { Loading (\%) }\end{array}$ & $\begin{array}{c}\text { Alcohol: } \\
\text { Fatty Acid } \\
\text { Molar Ratio }\end{array}$ & $\begin{array}{l}\text { Time } \\
\text { (h) }\end{array}$ & $\begin{array}{l}\text { Temp. } \\
\left({ }^{\circ} \mathrm{C}\right)\end{array}$ & & & \\
\hline $\begin{array}{c}\text { C. rugosa lipases } \\
\text { immobilized on } \\
\text { immobead } 150\end{array}$ & $\begin{array}{l}\text { Acutodesmus } \\
\text { obliquus oil }\end{array}$ & 15 & $3: 1$ & 8 & 50 & 95.36 & $5 / 90.07$ & [161] \\
\hline $\begin{array}{c}\text { C. rugosa lipase } \\
\text { immobilized lipase } \\
\text { on core-shell } \\
\text { structured } \\
\mathrm{Fe}_{3} \mathrm{O}_{4} @ \mathrm{MIL}- \\
100(\mathrm{Fe}) \\
\text { composites } \\
\end{array}$ & Soybean oil & 25 & $4: 1$ & 60 & 40 & 92.3 & $5 / 83.6$ & [162] \\
\hline $\begin{array}{l}\text { C. rugosa lipase } \\
\text { immobilized on } \\
\text { magnetic } \\
\mathrm{Fe}_{3} \mathrm{O}_{4} \text {-poly } \\
\text { (glycidyl } \\
\text { methacrylate-co- } \\
\text { methacrylic acid) } \\
\text { composite }\end{array}$ & Soybean oil & 25 & $4: 1$ & 54 & 40 & 92.8 & $5 / 79.4$ & [204] \\
\hline $\begin{array}{c}\text { C. rugosa } \\
\text { immobilized on } \\
\mathrm{Mg} \text { modified } \\
\mathrm{Fe}_{2} \mathrm{O}_{4} \\
\text { nanoparticles }\end{array}$ & $\begin{array}{l}\text { Brewers' spent } \\
\text { grains oil }\end{array}$ & 30 & $4: 1$ & 48 & 45 & 98 & $4 / 87$ & [173] \\
\hline $\begin{array}{c}\text { Eversa }^{\circledR} \text { Transform } \\
2.0 \text { (liquid lipase } \\
\text { from T. lanuginosus) }\end{array}$ & Palm oil & 0.2 & $4: 1$ & 24 & 40 & 97 & - & [205] \\
\hline $\begin{array}{c}\text { A. oryzae ST11 } \\
\text { lipase immobilized } \\
\text { on } \\
\text { polyacrylonitrile } \\
\text { coated magnetic } \\
\text { nanoparticles }\end{array}$ & Palm oil & 30 & $3: 1$ & 24 & 37 & 94.7 & $5 / 65$ & [163] \\
\hline $\begin{array}{l}\text { B. cepacia lipase } \\
\text { immobilized on } \\
\text { hydroxyapatite } \\
\text { coated magnetic } \\
\text { nanoparticle }\end{array}$ & WCO & & $7: 1$ & 48 & 40 & 98 & $4 / 82$ & [164] \\
\hline $\begin{array}{l}\text { B. cepacia lipase } \\
\text { immobilized on } \\
\text { mesoporous } \\
\text { silica/iron oxide } \\
\text { magnetic core-shell } \\
\text { nanoparticle }\end{array}$ & WCO & 36 & $6.2: 1$ & 25 & 34 & 92 & $3 / 81$ & [165] \\
\hline B. cepacia lipase & Sunflower oil & 10 & $3.4: 1$ & 1 & 50 & $>99$ & - & [206] \\
\hline A. grandidieri lipase & Sunflower oil & 25 & $2: 1$ & 96 & 40 & 95 & - & [166] \\
\hline $\begin{array}{c}\text { R. oryzae lipase } \\
\text { immobilized on } \\
\text { alginate-polyvinyl } \\
\text { alcohol }\end{array}$ & Sludge palm oil & 2 & $3: 1$ & & 40 & 91.3 & $15 />91$ & [167] \\
\hline $\begin{array}{l}\text { P. fluorescens lipase } \\
\text { immobilized onto } \\
\mathrm{Co}^{2+} \text {-chelated } \\
\text { magnetic } \\
\text { nanoparticles }\end{array}$ & WCO & 7.5 & $4: 1$ & 12 & 50 & 95 & $10 / 83$ & [168] \\
\hline $\begin{array}{l}\text { Immobilized } L \text {. } \\
\text { plantarum lipase }\end{array}$ & Olive oil & 5 & $6: 1$ & 2 & 37 & 81 & $4 />65 \%$ & [169] \\
\hline
\end{tabular}


Table 3. Cont.

\begin{tabular}{|c|c|c|c|c|c|c|c|c|}
\hline \multirow[b]{2}{*}{ Catalyst } & \multirow[b]{2}{*}{ Feedstock } & \multicolumn{4}{|c|}{ Reaction Conditions } & \multirow[b]{2}{*}{$\begin{array}{l}\text { Conversion/ } \\
\text { Biodiesel } \\
\text { Yield (\%) }\end{array}$} & \multirow[b]{2}{*}{$\begin{array}{c}\text { Time of } \\
\text { Reuse/Corresponding } \\
\text { Biodiesel Yield }\end{array}$} & \multirow[b]{2}{*}{ Ref. } \\
\hline & & $\begin{array}{c}\text { Catalyst } \\
\text { Loading (\%) }\end{array}$ & $\begin{array}{c}\text { Alcohol: } \\
\text { Fatty Acid } \\
\text { Molar Ratio }\end{array}$ & $\begin{array}{l}\text { Time } \\
\text { (h) }\end{array}$ & $\begin{array}{l}\text { Temp. } \\
\left({ }^{\circ} \mathrm{C}\right)\end{array}$ & & & \\
\hline $\begin{array}{c}\text { Lipase } \\
\text { immobilized on } \\
\text { graphene oxide }\end{array}$ & Karanja oil & 3 & $8: 1$ & 24 & 25 & 88 & - & [174] \\
\hline $\begin{array}{c}\text { Oreochromis } \\
\text { niloticus lipase }\end{array}$ & WCO & 30 kUnit & $4: 1$ & 28 & 45 & 96.5 & & [151] \\
\hline $\begin{array}{l}\text { A. terreus AH-F2 } \\
\text { lipase immobilized } \\
\text { on polydopamine } \\
\text { coated iron oxide }\end{array}$ & WCO & 10 & $6: 1$ & 30 & 37 & 92 & $5 />80$ & [170] \\
\hline $\begin{array}{l}\text { Steapsin lipase } \\
\text { immobilized on } \\
\text { waste-derived } \\
\text { activated carbon } \\
\text { support } \\
\end{array}$ & Rubber seed oil & 3 & $6: 1$ & 5 & 20 & 83.9 & $7 />77$ & [177] \\
\hline $\begin{array}{l}\text { Steapsin lipase } \\
\text { immobilized on } \\
\text { Immobead-350 }\end{array}$ & WCO & 14 & $4: 28$ & 14 & 40 & 88.33 & & [207] \\
\hline $\begin{array}{c}\text { Proteus sp. NH 2-2 } \\
\text { lipase }\end{array}$ & soybean oil & 0.5 & $4: 1$ & 36 & 40 & 91.5 & & [208] \\
\hline Garbage lipase & $\begin{array}{l}\text { Naganishia } \\
\text { liquefaciens } \\
\text { NITTS2 oil }\end{array}$ & 20 & $6.4: 1$ & 16 & 35 & 97.13 & & [209] \\
\hline $\begin{array}{l}\text { Lipase (from } \\
\text { porcine pancreas) } \\
\text { immobilized on } \\
\text { genipin } \\
\text { cross-linked } \\
\text { chitosan beads }\end{array}$ & WCO & 7.5 & $9: 1$ & 10 & 40 & 92.33 & $4 />80$ & [178] \\
\hline
\end{tabular}

Liquid lipase formulations or free lipases have been considered as a substitute for immobilized lipase for biodiesel production due to their high catalytic activity and significantly low cost (30 to 50 times lower) as compared to immobilized lipase $[183,210]$. Recent studies have demonstrated a promising use of several liquid lipases for biodiesel production such as C. antarctica lipase A [154] and liquid lipase formulations from T. lanuginosus (Eversa ${ }^{\circledR}$ Transform, Eversa ${ }^{\circledR}$ Transform 2.0, and NS-40116) $[195,205,211]$. The use of liquid lipase facilitates the homogenous reaction, thus overcoming the mass transfer limitation presented in the immobilized lipase-catalyzed reaction. However, liquid lipase is sensitive to the reaction environment. Studies have reported that high water content (from the feedstock and/or generated from the esterification of alcohol and fatty acid) not only promotes the reverse reaction but also negatively affects the lipase activity (including the formation of lipase-lipase aggregates in aqueous media), thus reducing the biodiesel production efficiency [183]. To address this obstacle, several adsorbents such as superabsorbent polymer, silica gel, alumina, and molecular sieve have been used to remove the water from the reaction mixture, enhancing the reaction efficiency [183,212,213].

Similarly, the type of acyl acceptor used also affects the lipase-catalyzed reaction. Studies have reported that lipase is deactivated using a high amount of methanol or ethanol, lowering the biodiesel yield [181,185]. In addition, the use of methanol or ethanol as an acyl acceptor for biodiesel production resulted in the formation of by-product glycerol [181]. This by-product also inhibits the activity of lipases, especially immobilized lipases because it can easily accumulate on the surface of immobilized lipases [181]. To address this obstacle, methyl acetate is proposed as another alternative acyl acceptor for biodiesel production [185]. The use of methyl acetate prevents the inhibition of lipase caused by methanol/ethanol and by-product glycerol (no glycerol produced in the reaction), thus 
enhancing the reaction rate [185]. Besides this method, ultrasounds [214,215] or very hydrophobic supports $[216,217]$ can be used as another approach to lower the negative effect of glycerol on the enzyme. Studies have reported that ultrasounds can stir the enzyme particles from the inside and avoid the formation of the glycerin/water phase $[215,218]$.

Another concern for each specific lipase in biodiesel production is associated with the oil source [218]. Fats/oils are a very heterogenous substrate, which are mainly comprised of triglycerides, low levels of mono and diglycerides, and some FFA [218]. Therefore, enzyme specificity affects the enzyme activity over each substrate [218]. To address this issue, the combination of different lipases (combi-lipase) has been proposed for biodiesel production [190,219]. There are several types of combi-lipase, which include co-immobilized lipases (different lipases immobilized on the same support), a mixture of individually immobilized lipases, and a mixture of free lipases [218]. Guan et al. [219] firstly reported the use of R. miehei lipase and P. cyclopium lipase mixture (in a liquid form) for biodiesel production from soybean oil. The result showed that the $R$. miehei lipase (individual enzyme) resulted in $68.5 \%$ biodiesel yield, but the yield increased to $95 \%$ when using the mixture of R. miehei and P. cyclopium lipases [219]. This was due to the use of lipases with different specificities $[218,219]$. In another study, the individual use of $R$. oryzae lipase and C. rugosa lipase resulted in $94.36 \%$ biodiesel yield at a reaction time of $9 \mathrm{~h}$ and $92.63 \%$ biodiesel yield at a reaction time of $30 \mathrm{~h}$, respectively [220]. However, the biodiesel yield reached $98.16 \%$ (at a reaction time of $6 \mathrm{~h}$ ) by using the mixture of both enzymes [220]. Similarly, various combilipases such as lipase cocktail (67\% C. antarctica lipase B and 33\% R. miehei lipase) [190]; immobilized C. rugosa and R. miehei lipases [175,202], co-immobilized R. miehei lipase and C. antarctica lipase B [193]; a mixture of $10 \%$ T. lanuginosus lipase, $75 \%$ C. antarctica lipase $\mathrm{B}$, and $15 \%$ R. miehei lipase [221]; a mixture of lipases from porcine and T. lanuginosus (in both liquid and immobilized forms) [222]; a mixture of immobilized C. rugosa and $R$. oryzae lipases [223], and co-immobilized C. rugosa and R. oryzae lipases [224,225] were also tested for biodiesel production. These combi-lipases showed a higher biodiesel yield than the individual enzymes $[175,190,193]$. Mixtures of the same enzyme immobilized using different protocols/support materials also affect the biodiesel yield. Toro et al. [226] immobilized the same lipase (T. lanuginosus lipase) on two different supports (Purolite ${ }^{\circledR}$ ECR1604 and Lewatit ${ }^{\circledR}$ VPOC1600) and used them for biodiesel production from palm olein. The biodiesel yield reached 70.3\% (for lipase immobilized on Purolite ${ }^{\circledR}$ ECR1604) and 78.2\% (for lipase immobilized on Lewatit ${ }^{\circledR}$ VPOC1600). Notably, the biodiesel yielded increased to $86.1 \%$ when the mixture of the two individually immobilized lipases was used [226]. This could be explained by the fact that the enzyme features (flexibility of their active site and their mechanism of action) can be modulated by changes in the immobilization protocol [172]. Consequently, the changes in the support feature influence the stability, activity, and specificity of the lipase [172,218].

Generally, although both immobilized and liquid lipases (individual lipases or combilipases) show effectiveness for converting oil into biodiesel, their industrial application is still limited due to the high cost of the enzyme as compared to chemical catalyst $[227,228]$. Therefore, further studies on lipase-catalyzed biodiesel production are still required to improve the efficiency and economic feasibility of the process.

\section{Catalyst Reusability}

For biodiesel conversion, the catalyst's effectiveness is not only determined by its catalytic activity but also its recoverability and reusability. Since homogenous catalysts cannot be reused for the next batch of production, heterogeneous catalysts play an important role in reducing production costs. Their recyclability not only lowers production costs but also maximizes environmental protection [229]. As compared to homogenous catalysts, one of the benefits of heterogeneous catalysts is that they can be reused several times. Furthermore, these catalysts may be regenerated or used for other purposes after losing their catalytic activity, such as construction materials, soil stabilizers, cement industries, and phosphate adsorbents [230]. 
Most of the bio-derived acid and alkali catalysts can be reused 4-7 times to yield biodiesel of $65-85 \%$ (Tables 1 and 2). da Luz Corrêa et al. [122] prepared sulfonated carbonbased catalysts from murumuru kernel shell and used them for FFA conversion. The first use of the catalyst resulted in $95.1 \%$, but the FFA conversion was reduced to $84.5 \%$ and $66.3 \%$ after the second and third catalyst reuses, respectively. The reusability of a solid base oxide catalyst derived from chicken eggshell was investigated by performing transesterification using the same catalyst for 10 cycles, and the yield was found to be marginally reduced after the seventh cycle, which may be due to catalyst pores being blocked, reducing reactant adsorption and desorption [49]. Kirubakaran and Arul [27] also investigated the reusability of a heterogeneous catalyst derived from eggshell. The catalyst could be reused five times to yield $85 \%$ biodiesel. After that, the biodiesel yield reduced significantly, suggesting that the catalyst's stability had deteriorated. This is due to the presence of active $\mathrm{Ca}(\mathrm{OH})_{2}$ phases which reacted partially with the homogenous mixture in the transesterification reaction. In comparison with solid bio-based acid and alkali catalysts, several immobilized lipases show better reusability. Several immobilized lipases can be reused for up to 20 cycles without loss of enzyme activity [181,185]. However, the use of immobilized lipase for biodiesel production is still under lab-scale investigation because of the high cost of the enzyme. Therefore, to be used for industrial biodiesel production, further studies are still required to improve the catalytic activity, stability, and reusability of bio-based catalysts. In addition, a pilot-scale investigation is also needed to evaluate the potential use of these catalysts for biodiesel production before being used for industrial applications.

\section{Environmental and Economic Evaluation}

Catalyst selection is one of the crucial issues in biodiesel production with the aim to minimize energy consumption, waste generation and treatment, and reduce production costs [185]. The use of bio-based catalysts (alkali catalysts, acid catalysts, and enzymes) lowers the environmental effect since these catalysts are derived from natural sources (plants, animals, or microorganisms). These catalysts are also easy to separate from the reaction mixture and reuse, reducing the generation of wastewater and chemical residues in the downstream process, especially the purification step. Consequently, the fee for the purification step and waste treatment can be reduced, lowering the production cost.

Several studies have also evaluated the economic feasibility of different biodiesel production processes $[231,232]$. The bio-derived alkali- and bio-derived acid-catalyzed processes are more economically feasible than the conventional process $\left(\mathrm{H}_{2} \mathrm{SO}_{4}\right.$ - or $\mathrm{KOH}$ catalyzed process) for biodiesel production since the cost of those catalysts (and total biodiesel production cost) is considered lower than that of conventional chemical catalysts $\left(\mathrm{KOH}\right.$ or $\left.\mathrm{H}_{2} \mathrm{SO}_{4}\right)$ [232-234]. Among these two processes, the alkali-catalyzed transesterification seems to be superior to the acid-catalyzed process because the former proceeds at a lower temperature, has a shorter reaction time, and requires a lower molar ratio of alcohol to oil as compared to the latter, as shown in Tables 1 and 2 [232,235]. In addition, the bio-based alkali and bio-based acid catalysts can be synthesized from the same natural source, but the synthesis of bio-based acid catalyst commonly requires one more step (sulfonation) [232]. Consequently, in some cases, the cost of bio-derived acid catalysts can be higher than that of bio-derived alkali catalysts. However, the cost of each specific catalyst depends on various factors including the source, synthesis method, and its reusability [232]. Therefore, it is difficult to compare the cost of all different types of catalysts. Different from bio-derived acid and alkali catalysts, the enzyme is expensive, especially the immobilized enzyme, making the lipase-catalyzed biodiesel production less competitive $[236,237]$. To reduce the enzyme cost, free lipase (or liquid lipase formulation) has been proposed for biodiesel production [183]. However, the reusability of liquid lipases is limited [183]. Therefore, the enzymatic process is still under investigation to improve its industrial application. Generally, among the three processes, the bio-derived alkali- and bio-derived acid-catalyzed processes are more economically feasible than the enzymatic process [236]. 
However, no individual studies have been conducted to compare the economic feasibility of the bio-derived acid-, bio-derived alkali-, and enzyme-catalyzed biodiesel production processes. Therefore, more studies are still required to evaluate and compare the economic feasibility of these processes.

\section{Future Prospects and Conclusions}

The use of biomass-derived catalysts has become a recent interest to make biodiesel production more sustainable. In addition, the use of these catalysts is promising to reduce the current high cost of biodiesel production, making biodiesel competitive with petrodiesel fuels. Research is therefore aimed to develop environmentally friendly, cost-effective, and efficient biomass-derived catalysts for biodiesel production. Consequently, different natural sources (animals, plants, microorganisms) have been used for synthesizing biobased catalysts including acid catalysts, alkali catalysts, and enzymes. The catalytic activity of these catalysts varies among them. The use of acid or alkali catalysts depends on the quality of the feedstock. Besides, enzymes can be used as an alternative to both acid and alkali catalysts for biodiesel production. These catalysts show their advantages and disadvantages when they are used for biodiesel production. These catalysts show promise for biodiesel production, but these investigations have been stopped at lab-scale investigations. More investigations on these catalysts are therefore needed, especially largescale investigations to prove the potential use of these catalysts for industrial biodiesel production.

Author Contributions: Conceptualization, H.C.N., H.C.O. and C.-H.S.; writing-original draft preparation, M.-L.N., H.-Y.J., S.-J.W. and H.C.N.; writing-review and editing, H.C.N., H.C.O. and C.-H.S. All authors have read and agreed to the published version of the manuscript.

Funding: This research received no external funding.

Conflicts of Interest: The authors declare no conflict of interest.

\section{References}

1. Z Živković, S.B.; Veljković, M.V.; Banković-Ilić, I.B.; Krstić, I.M.; Konstantinović, S.S.; Ilić, S.B.; Avramović, J.M.; Stamenković, O.S.; Veljković, V.B. Technological, technical, economic, environmental, social, human health risk, toxicological and policy considerations of biodiesel production and use. Renew. Sustain. Energy Rev. 2017, 79, 222-247. [CrossRef]

2. Nguyen, H.C.; Nguyen, N.T.; Su, C.-H.; Wang, F.-M.; Tran, T.N.; Liao, Y.-T.; Liang, S.-H. Biodiesel production from insects: From organic waste to renewable energy. Curr. Org. Chem. 2019, 23, 1499-1508. [CrossRef]

3. Nguyen, H.C.; Nguyen, M.L.; Liang, S.-H.; Su, C.-H.; Wang, F.-M. Switchable solvent-catalyzed direct transesterification of insect biomass for biodiesel production. BioEnergy Res. 2020, 13, 563-570. [CrossRef]

4. Nguyen, H.C.; Nguyen, M.L.; Wang, F.-M.; Juan, H.-Y.; Su, C.-H. Biodiesel production by direct transesterification of wet spent coffee grounds using switchable solvent as a catalyst and solvent. Bioresour. Technol. 2020, 296, 122334. [CrossRef] [PubMed]

5. Nguyen, H.C.; Wang, F.-M.; Dinh, K.K.; Pham, T.T.; Juan, H.-Y.; Nguyen, N.P.; Ong, H.C.; Su, C.-H. Microwave-assisted noncatalytic esterification of fatty acid for biodiesel production: A kinetic study. Energies 2020, 13, 2167. [CrossRef]

6. Nguyen, H.C.; Liang, S.-H.; Li, S.-Y.; Su, C.-H.; Chien, C.-C.; Chen, Y.-J.; Huong, D.T.M. Direct transesterification of black soldier fly larvae (Hermetia illucens) for biodiesel production. J. Taiwan Inst. Chem. Eng. 2018, 85, 165-169. [CrossRef]

7. Nguyen, H.C.; Pan, J.W.; Su, C.H.; Ong, H.C.; Chern, J.M.; Lin, J.Y. Sol-gel synthesized lithium orthosilicate as a reusable solid catalyst for biodiesel production. Int. J. Energy Res. 2021, 45, 6239-6249. [CrossRef]

8. Niju, S.; Meera, K.; Begum, S.; Anantharaman, N. Modification of egg shell and its application in biodiesel production. J. Saudi Chem. Soc. 2014, 18, 702-706. [CrossRef]

9. Su, C.-H.; Nguyen, H.; Pham, U.; Nguyen, M.; Juan, H.-Y. Biodiesel production from a novel nonedible feedstock, soursop (Annona muricata L.) seed oil. Energies 2018, 11, 2562. [CrossRef]

10. Mardhiah, H.H.; Ong, H.C.; Masjuki, H.; Lim, S.; Lee, H. A review on latest developments and future prospects of heterogeneous catalyst in biodiesel production from non-edible oils. Renew. Sustain. Energy Rev. 2017, 67, 1225-1236. [CrossRef]

11. Bhuiya, M.; Rasul, M.; Khan, M.; Ashwath, N.; Azad, A. Prospects of 2nd generation biodiesel as a sustainable fuel-Part: 1 selection of feedstocks, oil extraction techniques and conversion technologies. Renew. Sustain. Energy Rev. 2016, 55, 1109-1128. [CrossRef]

12. Yaşar, F. Biodiesel production via waste eggshell as a low-cost heterogeneous catalyst: Its effects on some critical fuel properties and comparison with $\mathrm{CaO}$. Fuel 2019, 255, 115828. [CrossRef] 
13. Lee, S.L.; Wong, Y.C.; Tan, Y.P.; Yew, S.Y. Transesterification of palm oil to biodiesel by using waste obtuse horn shell-derived CaO catalyst. Energy Convers. Manag. 2015, 93, 282-288. [CrossRef]

14. Teo, S.H.; Rashid, U.; Choong, S.T.; Taufiq-Yap, Y.H. Heterogeneous calcium-based bimetallic oxide catalyzed transesterification of Elaeis guineensis derived triglycerides for biodiesel production. Energy Convers. Manag. 2017, 141, 20-27. [CrossRef]

15. Gupta, A.R.; Rathod, V.K. Waste cooking oil and waste chicken eggshells derived solid base catalyst for the biodiesel production: Optimization and kinetics. Waste Manag. 2018, 79, 169-178. [CrossRef]

16. Boonyuen, S.; Smith, S.M.; Malaithong, M.; Prokaew, A.; Cherdhirunkorn, B.; Luengnaruemitchai, A. Biodiesel production by a renewable catalyst from calcined Turbo jourdani (Gastropoda: Turbinidae) shells. J. Clean. Prod. 2018, 177, 925-929. [CrossRef]

17. Lin, Y.-C.; Amesho, K.T.; Chen, C.-E.; Cheng, P.-C.; Chou, F.-C. A cleaner process for green biodiesel synthesis from waste cooking oil using recycled waste oyster shells as a sustainable base heterogeneous catalyst under the microwave heating system. Sustain. Chem. Pharm. 2020, 17, 100310. [CrossRef]

18. Trisupakitti, S.; Ketwong, C.; Senajuk, W.; Phukapak, C.; Wiriyaumpaiwong, S. Golden apple cherry snail shell as catalyst for heterogeneous transesterification of biodiesel. Braz. J. Chem. Eng. 2018, 35, 1283-1291. [CrossRef]

19. Konwar, L.; Boro, J.; Deka, D. Activated carbon supported cao from waste shells as a catalyst for biodiesel production. Energy Sources Part A 2018, 40, 601-607. [CrossRef]

20. Madhu, D.; Arora, R.; Sahani, S.; Singh, V.; Sharma, Y.C. Synthesis of high-quality biodiesel using feedstock and catalyst derived from fish wastes. J. Agric. Food Chem. 2017, 65, 2100-2109. [CrossRef]

21. Hadiyanto, H.; Afianti, A.H.; Navi'a, U.I.; Adetya, N.P.; Widayat, W.; Sutanto, H. The development of heterogeneous catalyst $\mathrm{C} / \mathrm{CaO} / \mathrm{NaOH}$ from waste of green mussel shell (Perna varidis) for biodiesel synthesis. J. Environ. Chem. Eng. 2017, 5, 4559-4563. [CrossRef]

22. Aitlaalim, A.; Ouanji, F.; Benzaouak, A.; Mahi, M.E.; Lotfi, E.M.; Kacimi, M.; Liotta, L.F. Utilization of waste grooved razor shell (grs) as a catalyst in biodiesel production from refined and waste cooking oils. Catalysts 2020, 10, 703. [CrossRef]

23. Niju, S.; Anushya, C.; Balajii, M. Process optimization for biodiesel production from Moringa oleifera oil using conch shells as heterogeneous catalyst. Environ. Prog. Sustain. Energy 2019, 38, e13015. [CrossRef]

24. Niju, S.; Rabia, R.; Devi, K.S.; Kumar, M.N.; Balajii, M. Modified Malleus malleus shells for biodiesel production from waste cooking oil: An optimization study using box-behnken design. Waste Biomass Valoriz. 2020, 11, 793-806. [CrossRef]

25. Volli, V.; Purkait, M.K.; Shu, C.-M. Preparation and characterization of animal bone powder impregnated fly ash catalyst for transesterification. Sci. Total Environ. 2019, 669, 314-321. [CrossRef]

26. Mansir, N.; Teo, S.H.; Rashid, U.; Saiman, M.I.; Tan, Y.P.; Alsultan, G.A.; Taufiq-Yap, Y.H. Modified waste egg shell derived bifunctional catalyst for biodiesel production from high FFA waste cooking oil. A review. Renew. Sustain. Energy Rev. 2018, 82, 3645-3655. [CrossRef]

27. Kirubakaran, M. Eggshell as heterogeneous catalyst for synthesis of biodiesel from high free fatty acid chicken fat and its working characteristics on a CI engine. J. Environ. Chem. Eng. 2018, 6, 4490-4503.

28. Gollakota, A.; Volli, V.; Shu, C.-M. Transesterification of waste cooking oil using pyrolysis residue supported eggshell catalyst. Sci. Total Environ. 2019, 661, 316-325. [CrossRef]

29. Goli, J.; Sahu, O. Development of heterogeneous alkali catalyst from waste chicken eggshell for biodiesel production. Renew. Energy 2018, 128, 142-154. [CrossRef]

30. Balajii, M.; Niju, S. A novel biobased heterogeneous catalyst derived from Musa acuminata peduncle for biodiesel productionProcess optimization using central composite design. Energy Convers. Manag. 2019, 189, 118-131. [CrossRef]

31. Mendonça, I.M.; Machado, F.L.; Silva, C.C.; Junior, S.D.; Takeno, M.L.; de Sousa Maia, P.J.; Manzato, L.; de Freitas, F.A. Application of calcined waste cupuaçu (Theobroma grandiflorum) seeds as a low-cost solid catalyst in soybean oil ethanolysis: Statistical optimization. Energy Convers. Manag. 2019, 200, 112095. [CrossRef]

32. Fan, M.; Wu, H.; Shi, M.; Zhang, P.; Jiang, P. Well-dispersive $\mathrm{K}_{2} \mathrm{OKCl}$ alkaline catalyst derived from waste banana peel for biodiesel synthesis. Green Energy Environ. 2019, 4, 322-327. [CrossRef]

33. Falowo, O.A.; Oloko-Oba, M.I.; Betiku, E. Biodiesel production intensification via microwave irradiation-assisted transesterification of oil blend using nanoparticles from elephant-ear tree pod husk as a base heterogeneous catalyst. Chem. Eng. Process. 2019, 140, 157-170. [CrossRef]

34. Oladipo, B.; Betiku, E. Optimization and kinetic studies on conversion of rubber seed (Hevea brasiliensis) oil to methyl esters over a green biowaste catalyst. J. Environ. Manag. 2020, 268, 110705. [CrossRef]

35. Nath, B.; Das, B.; Kalita, P.; Basumatary, S. Waste to value addition: Utilization of waste Brassica nigra plant derived novel green heterogeneous base catalyst for effective synthesis of biodiesel. J. Clean. Prod. 2019, 239, 118112. [CrossRef]

36. Fatimah, I.; Rubiyanto, D.; Taushiyah, A.; Najah, F.B.; Azmi, U.; Sim, Y.-L. Use of $\mathrm{ZrO}_{2}$ supported on bamboo leaf ash as a heterogeneous catalyst in microwave-assisted biodiesel conversion. Sustain. Chem. Pharm. 2019, 12, 100129. [CrossRef]

37. Nath, B.; Kalita, P.; Das, B.; Basumatary, S. Highly efficient renewable heterogeneous base catalyst derived from waste Sesamum indicum plant for synthesis of biodiesel. Renew. Energy 2020, 151, 295-310. [CrossRef]

38. Gohain, M.; Laskar, K.; Phukon, H.; Bora, U.; Kalita, D.; Deka, D. Towards sustainable biodiesel and chemical production: Multifunctional use of heterogeneous catalyst from littered Tectona grandis leaves. Waste Manag. 2020, 102, 212-221. [CrossRef] [PubMed] 
39. Kamel, D.A.; Farag, H.A.; Amin, N.K.; Zatout, A.A.; Fouad, Y.O. Utilization of Ficus carica leaves as a heterogeneous catalyst for production of biodiesel from waste cooking oil. Environ. Sci. Pollut. Res. 2019, 26, 32804-32814. [CrossRef]

40. John, M.; Abdullah, M.O.; Hua, T.Y.; Nolasco-Hipólito, C. Techno-economical and energy analysis of sunflower oil biodiesel synthesis assisted with waste ginger leaves derived catalysts. Renew. Energy 2021, 168, 815-828. [CrossRef]

41. Gohain, M.; Laskar, K.; Paul, A.K.; Daimary, N.; Maharana, M.; Goswami, I.K.; Hazarika, A.; Bora, U.; Deka, D. Carica papaya stem: A source of versatile heterogeneous catalyst for biodiesel production and C-C bond formation. Renew. Energy 2020, 147, 541-555. [CrossRef]

42. Gohain, M.; Devi, A.; Deka, D. Musa balbisiana Colla peel as highly effective renewable heterogeneous base catalyst for biodiesel production. Ind. Crops Prod. 2017, 109, 8-18. [CrossRef]

43. Naeem, M.M.; Al-Sakkari, E.G.; Boffito, D.C.; Gadalla, M.A.; Ashour, F.H. One-pot conversion of highly acidic waste cooking oil into biodiesel over a novel bio-based bi-functional catalyst. Fuel 2021, 283, 118914. [CrossRef]

44. Hazmi, B.; Rashid, U.; Taufiq-Yap, Y.H.; Ibrahim, M.L.; Nehdi, I.A. Supermagnetic nano-bifunctional catalyst from rice husk: Synthesis, characterization and application for conversion of used cooking oil to biodiesel. Catalysts 2020, 10, 225. [CrossRef]

45. Farooq, M.; Ramli, A.; Naeem, A.; Mahmood, T.; Ahmad, S.; Humayun, M.; Islam, M.G.U. Biodiesel production from date seed oil (Phoenix dactylifera L.) via egg shell derived heterogeneous catalyst. Chem. Eng. Res. Des. 2018, 132, 644-651. [CrossRef]

46. Putra, M.D.; Irawan, C.; Ristianingsih, Y.; Nata, I.F. A cleaner process for biodiesel production from waste cooking oil using waste materials as a heterogeneous catalyst and its kinetic study. J. Clean. Prod. 2018, 195, 1249-1258. [CrossRef]

47. Teo, S.H.; Islam, A.; Masoumi, H.R.F.; Taufiq-Yap, Y.H.; Janaun, J.; Chan, E.-S. Effective synthesis of biodiesel from Jatropha curcas oil using betaine assisted nanoparticle heterogeneous catalyst from eggshell of Gallus domesticus. Renew. Energy 2017, 111, 892-905. [CrossRef]

48. Pandit, P.R.; Fulekar, M. Biodiesel production from microalgal biomass using CaO catalyst synthesized from natural waste material. Renew. Energy 2019, 136, 837-845. [CrossRef]

49. Chingakham, C.; David, A.; Sajith, $\mathrm{V} . \mathrm{Fe}_{3} \mathrm{O}_{4}$ nanoparticles impregnated eggshell as a novel catalyst for enhanced biodiesel production. Chin. J. Chem. Eng. 2019, 27, 2835-2843. [CrossRef]

50. Marwaha, A.; Rosha, P.; Mohapatra, S.K.; Mahla, S.K.; Dhir, A. Biodiesel production from Terminalia bellerica using eggshell-based green catalyst: An optimization study with response surface methodology. Energy Rep. 2019, 5, 1580-1588. [CrossRef]

51. Ajala, E.O.; Ajala, M.A.; Odetoye, T.E.; Aderibigbe, F.A.; Osanyinpeju, H.O.; Ayanshola, M.A. Thermal modification of chicken eggshell as heterogeneous catalyst for palm kernel biodiesel production in an optimization process. Biomass Convers. Bior. 2020, 1-17. [CrossRef]

52. Lakshmi, S.B.A.V.S.; Pillai, N.S.; Mohamed, M.S.B.K.; Narayanan, A. Biodiesel production from rubber seed oil using calcined eggshells impregnated with $\mathrm{Al}_{2} \mathrm{O}_{3}$ as heterogeneous catalyst: A comparative study of $\mathrm{RSM}$ and ANN optimization. Braz. J. Chem. Eng. 2020, 37, 351-368.

53. Sai, B.A.; Subramaniapillai, N.; Mohamed, M.S.B.K.; Narayanan, A. Optimization of continuous biodiesel production from rubber seed oil (RSO) using calcined eggshells as heterogeneous catalyst. J. Environ. Chem. Eng. 2020, 8, 103603.

54. Pavlović, S.M.; Marinković, D.M.; Kostić, M.D.; Janković-Častvan, I.M.; Mojović, L.V.; Stanković, M.V.; Veljković, V.B. A $\mathrm{CaO}$ /zeolite-based catalyst obtained from waste chicken eggshell and coal fly ash for biodiesel production. Fuel 2020, 267, 117171. [CrossRef]

55. Peng, Y.-P.; Amesho, K.T.; Chen, C.-E.; Jhang, S.-R.; Chou, F.-C.; Lin, Y.-C. Optimization of biodiesel production from waste cooking oil using waste eggshell as a base catalyst under a microwave heating system. Catalysts 2018, 8, 81. [CrossRef]

56. Helwani, Z.; Ramli, M.; Saputra, E.; Putra, Y.L.; Simbolon, D.F.; Othman, M.R.; Idroes, R. Composite catalyst of palm mill fly ash-supported calcium oxide obtained from eggshells for transesterification of off-grade palm oil. Catalysts 2020, 10, 724. [CrossRef]

57. Kusmiyati, K.; Prasetyoko, D.; Murwani, S.; Nur Fadhilah, M.; Oetami, T.P.; Hadiyanto, H.; Widayat, W.; Budiman, A.; Roesyadi, A. Biodiesel production from Reutealis trisperma oil using KOH impregnated eggshell as a heterogeneous catalyst. Energies 2019, 12, 3714. [CrossRef]

58. Mansir, N.; Teo, S.H.; Ibrahim, M.L.; Hin, T.-Y.Y. Synthesis and application of waste egg shell derived CaO supported W-Mo mixed oxide catalysts for FAME production from waste cooking oil: Effect of stoichiometry. Energy Convers. Manag. 2017, 151, 216-226. [CrossRef]

59. Oladipo, A.S.; Ajayi, O.A.; Oladipo, A.A.; Azarmi, S.L.; Nurudeen, Y.; Atta, A.Y.; Ogunyemi, S.S. Magnetic recyclable eggshellbased mesoporous catalyst for biodiesel production from crude neem oil: Process optimization by central composite design and artificial neural network. C. R. Chim. 2018, 21, 684-695. [CrossRef]

60. Fayyazi, E.; Ghobadian, B.; van de Bovenkamp, H.H.; Najafi, G.; Hosseinzadehsamani, B.; Heeres, H.J.; Yue, J. Optimization of biodiesel production over chicken eggshell-derived $\mathrm{CaO}$ catalyst in a continuous centrifugal contactor separator. Ind. Eng. Chem. Res. 2018, 57, 12742-12755. [CrossRef]

61. Ngaosuwan, K.; Chaiyariyakul, W.; Inthong, O.; Kiatkittipong, W.; Wongsawaeng, D.; Assabumrungrat, S. La $2 \mathrm{O}_{3} / \mathrm{CaO}_{\mathrm{O}}$ catalyst derived from eggshells: Effects of preparation method and La content on textural properties and catalytic activity for transesterification. Catal. Commun. 2021, 149, 106247. [CrossRef] 
62. Tan, Y.H.; Abdullah, M.O.; Nolasco-Hipolito, C.; Zauzi, N.S.A. Application of RSM and Taguchi methods for optimizing the transesterification of waste cooking oil catalyzed by solid ostrich and chicken-eggshell derived CaO. Renew. Energy 2017, 114, 437-447. [CrossRef]

63. Helwani, Z.; Ramli, M.; Saputra, E.; Bahruddin, B.; Yolanda, D.; Fatra, W.; Idroes, G.M.; Muslem, M.; Mahlia, T.M.I.; Idroes, R. Impregnation of $\mathrm{CaO}$ from eggshell waste with magnetite as a solid catalyst $\left(\mathrm{Fe}_{3} \mathrm{O}_{4} / \mathrm{CaO}\right)$ for transesterification of palm oil off-grade. Catalysts 2020, 10, 164. [CrossRef]

64. Bharti, R.; Guldhe, A.; Kumar, D.; Singh, B. Solar irradiation assisted synthesis of biodiesel from waste cooking oil using calcium oxide derived from chicken eggshell. Fuel 2020, 273, 117778. [CrossRef]

65. Satya Lakshmi, S.B.A.V.; Niju, S.; Khadhar Mohamed, M.S.B.; Narayanan, A. Catalyst reusability and kinetic modeling of biodiesel produced from rubber seed oil. Energy Sources Part A 2020, 1-16. [CrossRef]

66. Graziottin, P.L.; Rosset, M.; dos Santos Lima, D.; Perez-Lopez, O.W. Transesterification of different vegetable oils using eggshells from various sources as catalyst. Vib. Spectrosc. 2020, 109, 103087. [CrossRef]

67. Foroutan, R.; Mohammadi, R.; Esmaeili, H.; Bektashi, F.M.; Tamjidi, S. Transesterification of waste edible oils to biodiesel using calcium oxide@ magnesium oxide nanocatalyst. Waste Manag. 2020, 105, 373-383. [CrossRef]

68. Palitsakun, S.; Koonkuer, K.; Topool, B.; Seubsai, A.; Sudsakorn, K. Transesterification of Jatropha oil to biodiesel using SrO catalysts modified with $\mathrm{CaO}$ from waste eggshell. Catal. Commun. 2021, 149, 106233. [CrossRef]

69. Khatibi, M.; Khorasheh, F.; Larimi, A. Biodiesel production via transesterification of canola oil in the presence of Na-K doped CaO derived from calcined eggshell. Renew. Energy 2021, 163, 1626-1636. [CrossRef]

70. Ahmad, S.; Chaudhary, S.; Pathak, V.V.; Kothari, R.; Tyagi, V. Optimization of direct transesterification of Chlorella pyrenoidosa catalyzed by waste egg shell based heterogenous nano-CaO catalyst. Renew. Energy 2020, 160, 86-97. [CrossRef]

71. Singh, T.S.; Verma, T.N. Biodiesel production from Momordica charantia (L.): Extraction and engine characteristics. Energy 2019, 189, 116198. [CrossRef]

72. Chowdhury, S.; Dhawane, S.H.; Jha, B.; Pal, S.; Sagar, R.; Hossain, A.; Halder, G. Biodiesel synthesis from transesterified Madhuca indica oil by waste egg shell-derived heterogeneous catalyst: Parametric optimization by Taguchi approach. Biomass Convers. Bior. 2019, 1-11. [CrossRef]

73. Borah, M.J.; Das, A.; Das, V.; Bhuyan, N.; Deka, D. Transesterification of waste cooking oil for biodiesel production catalyzed by Zn substituted waste egg shell derived CaO nanocatalyst. Fuel 2019, 242, 345-354. [CrossRef]

74. Rahman, W.U.; Fatima, A.; Anwer, A.H.; Athar, M.; Khan, M.Z.; Khan, N.A.; Halder, G. Biodiesel synthesis from eucalyptus oil by utilizing waste egg shell derived calcium based metal oxide catalyst. Process. Saf. Environ. Prot. 2019, 122, 313-319. [CrossRef]

75. Kolakoti, A.; Satish, G. Biodiesel production from low-grade oil using heterogeneous catalyst: An optimisation and ANN modelling. Aust. J. Mech. Eng. 2020, 1-13. [CrossRef]

76. Rahman, M. Valorization of harmful algae E. compressa for biodiesel production in presence of chicken waste derived catalyst. Renew. Energy 2018, 129, 132-140. [CrossRef]

77. Mansir, N.; Teo, S.H.; Rashid, U.; Taufiq-Yap, Y.H. Efficient waste Gallus domesticus shell derived calcium-based catalyst for biodiesel production. Fuel 2018, 211, 67-75. [CrossRef]

78. Krishnamurthy, K.; Sridhara, S.; Kumar, C.A. Optimization and kinetic study of biodiesel production from Hydnocarpus wightiana oil and dairy waste scum using snail shell CaO nano catalyst. Renew. Energy 2020, 146, 280-296. [CrossRef]

79. Gupta, J.; Agarwal, M. Preparation and characterization of highly active solid base catalyst from snail shell for biodiesel production. Biofuels 2019, 10, 315-324. [CrossRef]

80. Laskar, I.B.; Rajkumari, K.; Gupta, R.; Chatterjee, S.; Paul, B.; Rokhum, L. Waste snail shell derived heterogeneous catalyst for biodiesel production by the transesterification of soybean oil. RSC Adv. 2018, 8, 20131-20142. [CrossRef]

81. Marques Correia, L.; Cecilia, J.A.; Rodríguez-Castellón, E.; Cavalcante, C.L.; Vieira, R.S. Relevance of the physicochemical properties of calcined quail eggshell $(\mathrm{CaO})$ as a catalyst for biodiesel production. J. Chem. 2017, 2017. [CrossRef]

82. Adepoju, T.; Ibeh, M.; Babatunde, E.; Asquo, A. Methanolysis of CaO based catalyst derived from egg shell-snail shell-wood ash mixed for fatty acid methylester (FAME) synthesis from a ternary mixture of Irvingia gabonensis-Pentaclethra macrophylla-Elais guineensis oil blend: An application of simplex lattice and central composite design optimization. Fuel 2020, $275,117997$.

83. Pradhan, P.; Chakraborty, R. Optimal efficient biodiesel synthesis from used oil employing low-cost ram bone supported Cr catalyst: Engine performance and exhaust assessment. Energy 2018, 164, 35-45. [CrossRef]

84. AlSharifi, M.; Znad, H. Development of a lithium based chicken bone ( $\mathrm{Li}-\mathrm{Cb}$ ) composite as an efficient catalyst for biodiesel production. Renew. Energy 2019, 136, 856-864. [CrossRef]

85. AlSharifi, M.; Znad, H. Transesterification of waste canola oil by lithium/zinc composite supported on waste chicken bone as an effective catalyst. Renew. Energy 2020, 151, 740-749. [CrossRef]

86. Mamo, T.T.; Mekonnen, Y.S. Microwave-assisted biodiesel production from microalgae, scenedesmus species, using goat bonemade nano-catalyst. Appl. Biochem. Biotechnol. 2020, 190, 1147-1162. [CrossRef] [PubMed]

87. Ali, C.H.; Asif, A.H.; Iqbal, T.; Qureshi, A.S.; Kazmi, M.A.; Yasin, S.; Danish, M.; Mu, B.-Z. Improved transesterification of waste cooking oil into biodiesel using calcined goat bone as a catalyst. Energy Sources Part A 2018, 40, 1076-1083. [CrossRef]

88. Tan, Y.H.; Abdullah, M.O.; Kansedo, J.; Mubarak, N.M.; San Chan, Y.; Nolasco-Hipolito, C. Biodiesel production from used cooking oil using green solid catalyst derived from calcined fusion waste chicken and fish bones. Renew. Energy 2019, 139, 696-706. [CrossRef] 
89. Khan, H.M.; Iqbal, T.; Ali, C.H.; Javaid, A.; Cheema, I.I. Sustainable biodiesel production from waste cooking oil utilizing waste ostrich (Struthio camelus) bones derived heterogeneous catalyst. Fuel 2020, 277, 118091. [CrossRef]

90. Moawia, R.M.; Nasef, M.M.; Mohamed, N.H.; Ripin, A.; Zakeri, M. Biopolymer catalyst for biodiesel production by functionalisation of radiation grafted flax fibres with diethylamine under optimised conditions. Radiat. Phys. Chem. 2019, $164,108375$. [CrossRef]

91. Changmai, B.; Sudarsanam, P.; Rokhum, L. Biodiesel production using a renewable mesoporous solid catalyst. Ind. Crops Prod. 2020, 145, 111911. [CrossRef]

92. Zhao, C.; Yang, L.; Xing, S.; Luo, W.; Wang, Z.; Lv, P. Biodiesel production by a highly effective renewable catalyst from pyrolytic rice husk. J. Clean. Prod. 2018, 199, 772-780. [CrossRef]

93. Lani, N.S.; Ngadi, N.; Yahya, N.Y.; Abd Rahman, R. Synthesis, characterization and performance of silica impregnated calcium oxide as heterogeneous catalyst in biodiesel production. J. Clean. Prod. 2017, 146, 116-124. [CrossRef]

94. Arumugam, A.; Sankaranarayanan, P. Biodiesel production and parameter optimization: An approach to utilize residual ash from sugarcane leaf, a novel heterogeneous catalyst, from Calophyllum inophyllum oil. Renew. Energy 2020, 153, 1272-1282. [CrossRef]

95. Mutalib, A.A.A.; Ibrahim, M.L.; Matmin, J.; Kassim, M.F.; Mastuli, M.S.; Taufiq-Yap, Y.H.; Shohaimi, N.A.M.; Islam, A.; Tan, Y.H.; Kaus, N.H.M. $\mathrm{SiO}_{2}$-Rich sugar cane bagasse ash catalyst for transesterification of palm oil. BioEnergy Res. 2020, 13, 986-997. [CrossRef]

96. Abd Manaf, I.S.; Rahim, M.H.A.; Govindan, N.; Maniam, G.P. A first report on biodiesel production from Aglaia korthalsii seed oil using waste marine barnacle as a solid catalyst. Ind. Crops Prod. 2018, 125, 395-400. [CrossRef]

97. Balajii, M.; Niju, S. Banana peduncle-A green and renewable heterogeneous base catalyst for biodiesel production from Ceiba pentandra oil. Renew. Energy 2020, 146, 2255-2269. [CrossRef]

98. Lani, N.S.; Ngadi, N.; Inuwa, I.M. New route for the synthesis of silica-supported calcium oxide catalyst in biodiesel production. Renew. Energy 2020, 156, 1266-1277. [CrossRef]

99. Khan, H.M.; Iqbal, T.; Ali, C.H.; Yasin, S.; Jamil, F. Waste quail beaks as renewable source for synthesizing novel catalysts for biodiesel production. Renew. Energy 2020, 154, 1035-1043. [CrossRef]

100. Miladinović, M.R.; Zdujić, M.V.; Veljović, D.N.; Krstić, J.B.; Banković-Ilić, I.B.; Veljković, V.B.; Stamenković, O.S. Valorization of walnut shell ash as a catalyst for biodiesel production. Renew. Energy 2020, 147, 1033-1043. [CrossRef]

101. Lu, W.; Alam, M.A.; Wu, C.; Wang, Z.; Wei, H. Enhanced deacidification of acidic oil catalyzed by sulfonated granular activated carbon using microwave irradiation for biodiesel production. Chem. Eng. Process. 2019, 135, 168-174. [CrossRef]

102. Niu, S.; Ning, Y.; Lu, C.; Han, K.; Yu, H.; Zhou, Y. Esterification of oleic acid to produce biodiesel catalyzed by sulfonated activated carbon from bamboo. Energy Convers. Manag. 2018, 163, 59-65. [CrossRef]

103. Rocha, P.D.; Oliveira, L.S.; Franca, A.S. Sulfonated activated carbon from corn cobs as heterogeneous catalysts for biodiesel production using microwave-assisted transesterification. Renew. Energy 2019, 143, 1710-1716. [CrossRef]

104. Lokman, I.M.; Rashid, U.; Taufiq-Yap, Y.H. Meso-and macroporous sulfonated starch solid acid catalyst for esterification of palm fatty acid distillate. Arab. J. Chem. 2016, 9, 179-189. [CrossRef]

105. Shu, Q.; Zhang, Q.; Xu, G.; Nawaz, Z.; Wang, D.; Wang, J. Synthesis of biodiesel from cottonseed oil and methanol using a carbon-based solid acid catalyst. Fuel Process. Technol. 2009, 90, 1002-1008. [CrossRef]

106. Mendaros, C.M.; Go, A.W.; Nietes, W.J.T.; Gollem, B.E.J.O.; Cabatingan, L.K. Direct sulfonation of cacao shell to synthesize a solid acid catalyst for the esterification of oleic acid with methanol. Renew. Energy 2020, 152, 320-330. [CrossRef]

107. Wadood, A.; Rana, A.; Basheer, C.; Razzaq, S.A.; Farooq, W. In situ Transesterification of microalgae Parachlorella kessleri biomass using sulfonated rice husk solid catalyst at room temperature. BioEnergy Res. 2020, 13, 530-541. [CrossRef]

108. Ning, Y.; Niu, S. Preparation and catalytic performance in esterification of a bamboo-based heterogeneous acid catalyst with microwave assistance. Energy Convers. Manag. 2017, 153, 446-454. [CrossRef]

109. Flores, K.P.; Omega, J.L.O.; Cabatingan, L.K.; Go, A.W.; Agapay, R.C.; Ju, Y.-H. Simultaneously carbonized and sulfonated sugarcane bagasse as solid acid catalyst for the esterification of oleic acid with methanol. Renew. Energy 2019, 130, 510-523. [CrossRef]

110. Chellappan, S.; Aparna, K.; Chingakham, C.; Sajith, V.; Nair, V. Microwave assisted biodiesel production using a novel Brønsted acid catalyst based on nanomagnetic biocomposite. Fuel 2019, 246, 268-276. [CrossRef]

111. Behera, B.; Dey, B.; Balasubramanian, P. Algal biodiesel production with engineered biochar as a heterogeneous solid acid catalyst. Bioresour. Technol. 2020, 310, 123392. [CrossRef] [PubMed]

112. Syazwani, O.N.; Ibrahim, M.L.; Kanda, H.; Goto, M.; Taufiq-Yap, Y. Esterification of high free fatty acids in supercritical methanol using sulfated angel wing shells as catalyst. J. Supercrit. Fluids 2017, 124, 1-9. [CrossRef]

113. Endut, A.; Abdullah, S.H.Y.S.; Hanapi, N.H.M.; Hamid, S.H.A.; Lananan, F.; Kamarudin, M.K.A.; Umar, R.; Juahir, H.; Khatoon, H. Optimization of biodiesel production by solid acid catalyst derived from coconut shell via response surface methodology. Int. Biodeterior. Biodegrad. 2017, 124, 250-257. [CrossRef]

114. Mardhiah, H.H.; Ong, H.C.; Masjuki, H.; Lim, S.; Pang, Y.L. Investigation of carbon-based solid acid catalyst from Jatropha curcas biomass in biodiesel production. Energy Convers. Manag. 2017, 144, 10-17. [CrossRef]

115. Ibrahim, N.A.; Rashid, U.; Taufiq-Yap, Y.H.; Yaw, T.C.S.; Ismail, I. Synthesis of carbonaceous solid acid magnetic catalyst from empty fruit bunch for esterification of palm fatty acid distillate (PFAD). Energy Convers. Manag. 2019, 195, 480-491. [CrossRef] 
116. Sangar, S.K.; Lan, C.S.; Razali, S.; Farabi, M.A.; Taufiq-Yap, Y.H. Methyl ester production from palm fatty acid distillate (PFAD) using sulfonated cow dung-derived carbon-based solid acid catalyst. Energy Convers. Manag. 2019, 196, 1306-1315. [CrossRef]

117. Syazwani, O.N.; Rashid, U.; Mastuli, M.S.; Taufiq-Yap, Y.H. Esterification of palm fatty acid distillate (PFAD) to biodiesel using bi-functional catalyst synthesized from waste angel wing shell (Cyrtopleura costata). Renew. Energy 2019, 131, 187-196. [CrossRef]

118. Thushari, I.; Babel, S. Biodiesel production from waste palm cooking oil using solid acid catalyst derived from coconut meal residue. Waste Biomass Valoriz. 2020, 11, 4941-4956. [CrossRef]

119. Naik, B.D.; Udayakumar, M. Optimization and characterization studies on the production of bio-diesel from WSO using carbon catalyst derived from coconut meal residue. Energy Sources Part A 2019, 1-16. [CrossRef]

120. Asikin-Mijan, N.; AbdulKareem-Alsultan, G.; Izham, S.M.; Taufiq-Yap, Y. Biodiesel production via simultaneous esterification and transesterification of chicken fat oil by mesoporous sulfated Ce supported activated carbon. Biomass Bioenergy 2020, 141, 105714.

121. Quah, R.V.; Tan, Y.H.; Mubarak, N.; Kansedo, J.; Khalid, M.; Abdullah, E.; Abdullah, M.O. Magnetic biochar derived from waste palm kernel shell for biodiesel production via sulfonation. Waste Manag. 2020, 118, 626-636. [CrossRef]

122. Da Luz Corrêa, A.P.; Bastos, R.R.C.; da Rocha Filho, G.N.; Zamian, J.R.; da Conceição, L.R.V. Preparation of sulfonated carbonbased catalysts from murumuru kernel shell and their performance in the esterification reaction. RSC Adv. 2020, 10, 20245-20256. [CrossRef]

123. Bastos, R.R.C.; da Luz Corrêa, A.P.; da Luz, P.T.S.; da Rocha Filho, G.N.; Zamian, J.R.; da Conceição, L.R.V. Optimization of biodiesel production using sulfonated carbon-based catalyst from an amazon agro-industrial waste. Energy Convers. Manag. 2020, 205, 112457. [CrossRef]

124. Chellappan, S.; Nair, V.; Sajith, V.; Aparna, K. Synthesis, optimization and characterization of biochar based catalyst from sawdust for simultaneous esterification and transesterification. Chin. J. Chem. Eng. 2018, 26, 2654-2663. [CrossRef]

125. Wang, W.; Lu, P.; Tang, H.; Ma, Y.; Yang, X. A Zanthoxylum bungeanum seed oil-based carbon solid acid catalyst for the production of biodiesel. New J. Chem. 2017, 41, 9256-9261. [CrossRef]

126. Akinfalabi, S.-I.; Rashid, U.; Shean, T.Y.C.; Nehdi, I.A.; Sbihi, H.M.; Gewik, M.M. Esterification of palm fatty acid distillate for biodiesel production catalyzed by synthesized kenaf seed cake-based sulfonated catalyst. Catalysts 2019, 9, 482. [CrossRef]

127. Rashid, U.; Soltani, S.; Yaw Choong, T.S.; Nehdi, I.A.; Ahmad, J.; Ngamcharussrivichai, C. Palm biochar-based sulphated zirconium $\left(\mathrm{Zr}-\mathrm{AC}-\mathrm{HSO}_{3}\right.$ ) catalyst for methyl ester production from palm fatty acid distillate. Catalysts 2019, 9, 1029. [CrossRef]

128. Lim, S.; Pang, Y.L.; Shuit, S.H.; Wong, K.H.; Leong, C.K. Synthesis and characterization of monk fruit seed (Siraitia grosvenorii)based heterogeneous acid catalyst for biodiesel production through esterification process. Int. J. Energy Res. 2020, 44, 9454-9465. [CrossRef]

129. Rashid, U.; Ahmad, J.; Ibrahim, M.L.; Nisar, J.; Hanif, M.A.; Shean, T.Y.C. Single-pot synthesis of biodiesel using efficient sulfonated-derived tea waste-heterogeneous catalyst. Materials 2019, 12, 2293. [CrossRef]

130. Ogbu, I.M.; Ajiwe, V.I.E.; Okoli, C.P. Performance evaluation of carbon-based heterogeneous acid catalyst derived from Hura crepitans seed pod for esterification of high FFA vegetable oil. BioEnergy Res. 2018, 11, 772-783. [CrossRef]

131. Choksi, H.; Pandian, S.; Gandhi, Y.H.; Deepalakshmi, S. Studies on production of biodiesel from Madhuca indica oil using a catalyst derived from cotton stalk. Energy Sources Part A 2019, 1-10. [CrossRef]

132. Bora, A.P.; Dhawane, S.H.; Anupam, K.; Halder, G. Biodiesel synthesis from Mesua ferrea oil using waste shell derived carbon catalyst. Renew. Energy 2018, 121, 195-204. [CrossRef]

133. Lim, S.; Yap, C.Y.; Pang, Y.L.; Wong, K.H. Biodiesel synthesis from oil palm empty fruit bunch biochar derived heterogeneous solid catalyst using 4-benzenediazonium sulfonate. J. Hazard. Mater. 2020, 390, 121532. [CrossRef]

134. Ibrahim, S.F.; Asikin-Mijan, N.; Ibrahim, M.L.; Abdulkareem-Alsultan, G.; Izham, S.M.; Taufiq-Yap, Y. Sulfonated functionalization of carbon derived corncob residue via hydrothermal synthesis route for esterification of palm fatty acid distillate. Energy Convers. Manag. 2020, 210, 112698. [CrossRef]

135. Thushari, I.; Babel, S. Sustainable utilization of waste palm oil and sulfonated carbon catalyst derived from coconut meal residue for biodiesel production. Bioresour. Technol. 2018, 248, 199-203. [CrossRef]

136. Agapay, R.C.; Liu, H.-C.; Ju, Y.-H.; Go, A.W.; Angkawijaya, A.E.; Nguyen, P.L.T.; Truong, C.T.; Quijote, K.L. Synthesis and initial evaluation of solid acid catalyst derived from spent coffee grounds for the esterification of oleic acid and methanol. Waste Biomass Valoriz. 2021, 1-11. [CrossRef]

137. Li, N.; Zhang, X.-L.; Zheng, X.-C.; Wang, G.-H.; Wang, X.-Y.; Zheng, G.-P. Efficient synthesis of ethyl levulinate fuel additives from levulinic acid catalyzed by sulfonated pine needle-derived carbon. Catal. Surv. Asia 2019, 23, 171-180. [CrossRef]

138. Rana, A.; Alghazal, M.S.; Alsaeedi, M.M.; Bakdash, R.S.; Basheer, C.; Al-Saadi, A.A. Preparation and characterization of biomass carbon-based solid acid catalysts for the esterification of marine algae for biodiesel production. BioEnergy Res. 2019, 12, 433-442. [CrossRef]

139. Malani, R.S.; Sardar, H.; Malviya, Y.; Goyal, A.; Moholkar, V.S. Ultrasound-intensified biodiesel production from mixed nonedible oil feedstock using heterogeneous acid catalyst supported on rubber de-oiled cake. Ind. Eng. Chem. Res. 2018, 57, 14926-14938. [CrossRef]

140. Zhang, F.; Tian, X.; Shah, M.; Yang, W. Synthesis of magnetic carbonaceous acids derived from hydrolysates of Jatropha hulls for catalytic biodiesel production. RSC Adv. 2017, 7, 11403-11413. [CrossRef] 
141. Hussein, M.F.; El Naga, A.O.A.; El Saied, M.; AbuBaker, M.M.; Shaban, S.A.; El Kady, F.Y. Potato peel waste-derived carbon-based solid acid for the esterification of oleic acid to biodiesel. Environ. Technol. Innov. 2021, 21, 101355. [CrossRef]

142. Deeba, F.; Kumar, B.; Arora, N.; Singh, S.; Kumar, A.; Han, S.S.; Negi, Y.S. Novel bio-based solid acid catalyst derived from waste yeast residue for biodiesel production. Renew. Energy 2020, 159, 127-139. [CrossRef]

143. Bureros, G.M.A.; Tanjay, A.A.; Cuizon, D.E.S.; Go, A.W.; Cabatingan, L.K.; Agapay, R.C.; Ju, Y.-H. Cacao shell-derived solid acid catalyst for esterification of oleic acid with methanol. Renew. Energy 2019, 138, 489-501. [CrossRef]

144. Thushari, I.; Babel, S.; Samart, C. Biodiesel production in an autoclave reactor using waste palm oil and coconut coir husk derived catalyst. Renew. Energy 2019, 134, 125-134. [CrossRef]

145. Sandouqa, A.; Al-Hamamre, Z.; Asfar, J. Preparation and performance investigation of a lignin-based solid acid catalyst manufactured from olive cake for biodiesel production. Renew. Energy 2019, 132, 667-682. [CrossRef]

146. Akinfalabi, S.-I.; Rashid, U.; Yunus, R.; Taufiq-Yap, Y.H. Synthesis of biodiesel from palm fatty acid distillate using sulfonated palm seed cake catalyst. Renew. Energy 2017, 111, 611-619. [CrossRef]

147. Akhabue, C.E.; Osa-Benedict, E.O.; Oyedoh, E.A.; Otoikhian, S.K. Development of a bio-based bifunctional catalyst for simultaneous esterification and transesterification of neem seed oil: Modeling and optimization studies. Renew. Energy 2020, 152, 724-735. [CrossRef]

148. Arumugamurthy, S.S.; Sivanandi, P.; Pandian, S.; Choksi, H.; Subramanian, D. Conversion of a low value industrial waste into biodiesel using a catalyst derived from brewery waste: An activation and deactivation kinetic study. Waste Manag. 2019, 100, 318-326. [CrossRef]

149. Nguyen, H.C.; Nguyen, M.L.; Wang, F.-M.; Liang, S.-H.; Bui, T.L.; Ha, H.H.; Su, C.-H. Using switchable solvent as a solvent and catalyst for in situ transesterification of spent coffee grounds for biodiesel synthesis. Bioresour. Technol. 2019, $289,121770$. [CrossRef]

150. Ranganathan, S.V.; Narasimhan, S.L.; Muthukumar, K. An overview of enzymatic production of biodiesel. Bioresour. Technol. 2008, 99, 3975-3981. [CrossRef]

151. Patchimpet, J.; Simpson, B.K.; Sangkharak, K.; Klomklao, S. Optimization of process variables for the production of biodiesel by transesterification of used cooking oil using lipase from Nile tilapia viscera. Renew. Energy 2020, 153, 861-869. [CrossRef]

152. Mounguengui, R.W.M.; Brunschwig, C.; Baréa, B.; Villeneuve, P.; Blin, J. Are plant lipases a promising alternative to catalyze transesterification for biodiesel production? Progress Energy Combust. Sci. 2013, 39, 441-456. [CrossRef]

153. Zhang, H.; Liu, T.; Zhu, Y.; Hong, L.; Li, T.; Wang, X.; Fu, Y. Lipases immobilized on the modified polyporous magnetic cellulose support as an efficient and recyclable catalyst for biodiesel production from Yellow horn seed oil. Renew. Energy 2020, 145, 1246-1254. [CrossRef]

154. Guo, J.; Sun, S.; Liu, J. Conversion of waste frying palm oil into biodiesel using free lipase A from Candida antarctica as a novel catalyst. Fuel 2020, 267, 117323. [CrossRef]

155. Zhou, Y.; Li, K.; Sun, S. Simultaneous esterification and transesterification of waste phoenix seed oil with a high free fatty acid content using a free lipase catalyst to prepare biodiesel. Biomass Bioenergy 2021, 144, 105930. [CrossRef]

156. Sendzikiene, E.; Santaraite, M.; Makareviciene, V. Lipase-catalysed in situ transesterification of waste rapeseed oil to produce diesel-biodiesel blends. Processes 2020, 8, 1118. [CrossRef]

157. Aguieiras, E.C.; de Barros, D.S.; Fernandez-Lafuente, R.; Freire, D.M. Production of lipases in cottonseed meal and application of the fermented solid as biocatalyst in esterification and transesterification reactions. Renew. Energy 2019, 130, 574-581. [CrossRef]

158. Moreira, K.d.S.; de Oliveira, A.L.; Júnior, L.S.d.M.; Monteiro, R.R.; da Rocha, T.N.; Menezes, F.L.; Fechine, L.M.; Denardin, J.C.; Michea, S.; Freire, R.M. Lipase from Rhizomucor miehei immobilized on magnetic nanoparticles: Performance in fatty acid ethyl ester (faee) optimized production by the taguchi method. Front. Bioeng. Biotechnol. 2020, 8, 693. [CrossRef] [PubMed]

159. Kumar, D.; Das, T.; Giri, B.S.; Rene, E.R.; Verma, B. Biodiesel production from hybrid non-edible oil using bio-support beads immobilized with lipase from Pseudomonas cepacia. Fuel 2019, 255, 115801. [CrossRef]

160. Kumar, D.; Das, T.; Giri, B.S.; Verma, B. Optimization of biodiesel synthesis from nonedible oil using immobilized bio-support catalysts in jacketed packed bed bioreactor by response surface methodology. J. Clean. Prod. 2020, 244, 118700. [CrossRef]

161. Guldhe, A.; Singh, P.; Renuka, N.; Bux, F. Biodiesel synthesis from wastewater grown microalgal feedstock using enzymatic conversion: A greener approach. Fuel 2019, 237, 1112-1118. [CrossRef]

162. Xie, W.; Huang, M. Enzymatic production of biodiesel using immobilized lipase on core-shell structured Fe $\mathrm{O}_{4} @ \mathrm{MIL}-100(\mathrm{Fe})$ composites. Catalysts 2019, 9, 850. [CrossRef]

163. Paitaid, P.; Aran, H. Magnetic cross-linked enzyme aggregates of Aspergillus oryzae ST11 lipase using polyacrylonitrile coated magnetic nanoparticles for biodiesel production. Appl. Biochem. Biotechnol. 2020, 190, 1319-1332. [CrossRef] [PubMed]

164. Yang, H.; Zhang, W. Surfactant imprinting hyperactivated immobilized lipase as efficient biocatalyst for biodiesel production from waste cooking oil. Catalysts 2019, 9, 914. [CrossRef]

165. Khoobbakht, G.; Kheiralipour, K.; Yuan, W.; Seifi, M.R.; Karimi, M. Desirability function approach for optimization of enzymatic transesterification catalyzed by lipase immobilized on mesoporous magnetic nanoparticles. Renew. Energy 2020, 158, 253-262. [CrossRef]

166. Kouteu, P.A.N.; Blin, J.1.; Baréa, B.; Barouh, N.; Villeneuve, P. Solvent-free biodiesel production catalyzed by crude lipase powder from seeds: Effects of alcohol polarity, glycerol, and thermodynamic water activity. J. Agric. Food Chem. 2017, 65, 8683-8690. [CrossRef] [PubMed] 
167. Muanruksa, P.; Kaewkannetra, P. Combination of fatty acids extraction and enzymatic esterification for biodiesel production using sludge palm oil as a low-cost substrate. Renew. Energy 2020, 146, 901-906. [CrossRef]

168. Wang, J.; Li, K.; He, Y.; Wang, Y.; Han, X.; Yan, Y. Enhanced performance of lipase immobilized onto Co ${ }^{2+}$-chelated magnetic nanoparticles and its application in biodiesel production. Fuel 2019, 255, 115794. [CrossRef]

169. Khan, I.; Ganesan, R.; Dutta, J.R. Probiotic lipase derived from Lactobacillus plantarum and Lactobacillus brevis for biodiesel production from waste cooking olive oil: An alternative feedstock. Int. J. Green Energy 2020, 17, 62-70. [CrossRef]

170. Touqeer, T.; Mumtaz, M.W.; Mukhtar, H.; Irfan, A.; Akram, S.; Shabbir, A.; Rashid, U.; Nehdi, I.A.; Choong, T.S.Y. Fe ${ }_{3} \mathrm{O}_{4}-$ PDA-Lipase as surface functionalized nano biocatalyst for the production of biodiesel using waste cooking oil as feedstock: Characterization and process optimization. Energies 2020, 13, 177. [CrossRef]

171. Amini, Z.; Ilham, Z.; Ong, H.C.; Mazaheri, H.; Chen, W.-H. State of the art and prospective of lipase-catalyzed transesterification reaction for biodiesel production. Energy Convers. Manag. 2017, 141, 339-353. [CrossRef]

172. Tacias-Pascacio, V.G.; Virgen-Ortíz, J.J.; Jiménez-Pérez, M.; Yates, M.; Torrestiana-Sanchez, B.; Rosales-Quintero, A.; FernandezLafuente, R. Evaluation of different lipase biocatalysts in the production of biodiesel from used cooking oil: Critical role of the immobilization support. Fuel 2017, 200, 1-10. [CrossRef]

173. Iuliano, M.; Sarno, M.; De Pasquale, S.; Ponticorvo, E. Candida rugosa lipase for the biodiesel production from renewable sources. Renew. Energy 2020, 162, 124-133. [CrossRef]

174. Kumar, R.; Pal, P. Lipase immobilized graphene oxide biocatalyst assisted enzymatic transesterification of Pongamia pinnata (Karanja) oil and downstream enrichment of biodiesel by solar-driven direct contact membrane distillation followed by ultrafiltration. Fuel Process. Technol. 2021, 211, 106577. [CrossRef]

175. Binhayeeding, N.; Klomklao, S.; Prasertsan, P.; Sangkharak, K. Improvement of biodiesel production using waste cooking oil and applying single and mixed immobilised lipases on polyhydroxyalkanoate. Renew. Energy 2020, 162, 1819-1827. [CrossRef]

176. Sarno, M.; Iuliano, M. Highly active and stable $\mathrm{Fe}_{3} \mathrm{O}_{4} / \mathrm{Au}$ nanoparticles supporting lipase catalyst for biodiesel production from waste tomato. Appl. Surf. Sci. 2019, 474, 135-146. [CrossRef]

177. Dhawane, S.H.; Kumar, T.; Halder, G. Insight into biodiesel synthesis using biocatalyst designed through lipase immobilization onto waste derived microporous carbonaceous support. Process. Saf. Environ. Prot. 2019, 124, 231-239. [CrossRef]

178. Khan, N.; Maseet, M.; Basir, S.F. Synthesis and characterization of biodiesel from waste cooking oil by lipase immobilized on genipin cross-linked chitosan beads: A green approach. Int. J. Green Energy 2020, 17, 84-93. [CrossRef]

179. Moreira, K.S.; Moura Junior, L.S.; Monteiro, R.R.; de Oliveira, A.L.; Valle, C.P.; Freire, T.M.; Fechine, P.; de Souza, M.; FernandezLorente, G.; Guisan, J.M. Optimization of the production of enzymatic biodiesel from residual babassu oil (Orbignya sp.) via RSM. Catalysts 2020, 10, 414. [CrossRef]

180. Mukherjee, S.; Ghosh, M. Studies on performance evaluation of a green plasticizer made by enzymatic esterification of furfuryl alcohol and castor oil fatty acid. Carbohydr. Polym. 2017, 157, 1076-1084. [CrossRef]

181. Nguyen, H.C.; Liang, S.-H.; Doan, T.T.; Su, C.-H.; Yang, P.-C. Lipase-catalyzed synthesis of biodiesel from black soldier fly (Hermetica illucens): Optimization by using response surface methodology. Energy Convers. Manag. 2017, 145, 335-342. [CrossRef]

182. Li, J.; Zhang, J.; Shen, S.; Zhang, B.; William, W.Y. Magnetic responsive Thermomyces lanuginosus lipase for biodiesel synthesis. Mater. Today Commun. 2020, 24, 101197. [CrossRef] [PubMed]

183. Nguyen, H.C.; Huong, D.T.M.; Juan, H.-Y.; Su, C.-H.; Chien, C.-C. Liquid lipase-catalyzed esterification of oleic acid with methanol for biodiesel production in the presence of superabsorbent polymer: Optimization by using response surface methodology. Energies 2018, 11, 1085. [CrossRef]

184. Ren, H.; Li, Y.; Du, W.; Liu, D. Free lipase-catalyzed esterification of oleic acid for fatty acid ethyl ester preparation with response surface optimization. J. Am. Oil Chem. Soc. 2013, 90, 73-79. [CrossRef]

185. Chang, M.Y.; Chan, E.-S.; Song, C.P. Biodiesel production catalysed by low-cost liquid enzyme Eversa ${ }^{\circledR}$ Transform 2.0 : Effect of free fatty acid content on lipase methanol tolerance and kinetic model. Fuel 2021, 283, 119266. [CrossRef]

186. Monteiro, R.R.; Arana-Peña, S.; da Rocha, T.N.; Miranda, L.P.; Berenguer-Murcia, Á.; Tardioli, P.W.; dos Santos, J.C.; FernandezLafuente, R. Liquid lipase preparations designed for industrial production of biodiesel. Is it really an optimal solution? Renew. Energy 2021, 164, 1566-1587. [CrossRef]

187. Cavali, M.; Bueno, A.; Fagundes, A.P.; Priamo, W.L.; Bilibio, D.; Mibielli, G.M.; Wancura, J.H.; Bender, J.P.; Oliveira, J.V. Liquid lipase-mediated production of biodiesel from agroindustrial waste. Biocatal. Agric. Biotechnol. 2020, 30, 101864. [CrossRef]

188. Giacometti, J.; Giacometti, F.; Milin, Č.; Vasić-Rački, Đ. Kinetic characterisation of enzymatic esterification in a solvent system: Adsorptive control of water with molecular sieves. J. Mol. Catal. B Enzym. 2001, 11, 921-928. [CrossRef]

189. Gu, J.; Xin, Z.; Meng, X.; Sun, S.; Qiao, Q.; Deng, H. Studies on biodiesel production from DDGS-extracted corn oil at the catalysis of Novozym 435/super absorbent polymer. Fuel 2015, 146, 33-40. [CrossRef]

190. Nguyen, H.C.; Liang, S.-H.; Chen, S.-S.; Su, C.-H.; Lin, J.-H.; Chien, C.-C. Enzymatic production of biodiesel from insect fat using methyl acetate as an acyl acceptor: Optimization by using response surface methodology. Energy Convers. Manag. 2018, 158, 168-175. [CrossRef]

191. Martins, A.B.; Schein, M.F.; Friedrich, J.L.; Fernandez-Lafuente, R.; Ayub, M.A.; Rodrigues, R.C. Ultrasound-assisted butyl acetate synthesis catalyzed by Novozym 435: Enhanced activity and operational stability. Ultrason. Sonochem. 2013, 20, 1155-1160. [CrossRef] [PubMed] 
192. Paludo, N.; Alves, J.S.; Altmann, C.; Ayub, M.A.; Fernandez-Lafuente, R.; Rodrigues, R.C. The combined use of ultrasound and molecular sieves improves the synthesis of ethyl butyrate catalyzed by immobilized Thermomyces lanuginosus lipase. Ultrason. Sonochem. 2015, 22, 89-94. [CrossRef] [PubMed]

193. Poppe, J.K.; Garcia-Galan, C.; Matte, C.R.; Fernandez-Lafuente, R.; Rodrigues, R.C.; Ayub, M.A.Z. Optimization of synthesis of fatty acid methyl esters catalyzed by lipase B from Candida antarctica immobilized on hydrophobic supports. J. Mol. Catal. B Enzym. 2013, 94, 51-56. [CrossRef]

194. Graebin, N.G.; Martins, A.B.; Lorenzoni, A.S.; Garcia-Galan, C.; Fernandez-Lafuente, R.; Ayub, M.A.; Rodrigues, R.C. Immobilization of lipase B from Candida antarctica on porous styrene-divinylbenzene beads improves butyl acetate synthesis. Biotechnol. Prog. 2012, 28, 406-412. [CrossRef]

195. Arana-Peña, S.; Carballares, D.; Berenguer-Murcia, Á.; Alcántara, A.R.; Rodrigues, R.C.; Fernandez-Lafuente, R. One pot use of combilipases for full modification of oils and fats: Multifunctional and heterogeneous substrates. Catalysts 2020, 10, 605. [CrossRef]

196. Guan, F.; Peng, P.; Wang, G.; Yin, T.; Peng, Q.; Huang, J.; Guan, G.; Li, Y. Combination of two lipases more efficiently catalyzes methanolysis of soybean oil for biodiesel production in aqueous medium. Process. Biochem. 2010, 45, 1677-1682. [CrossRef]

197. Rocha, T.G.; Pedro, H.d.L.; de Souza, M.C.; Monteiro, R.R.; dos Santos, J.C. Lipase cocktail for optimized biodiesel production of free fatty acids from residual chicken oil. Catal. Lett. 2021, 151, 1155-1166. [CrossRef]

198. Zeng, L.; He, Y.; Jiao, L.; Li, K.; Yan, Y. Preparation of biodiesel with liquid synergetic lipases from rapeseed oil deodorizer distillate. Appl. Biochem. Biotechnol. 2017, 183, 778-791. [CrossRef]

199. Sangkharak, K.; Mhaisawat, S.; Rakkan, T.; Paichid, N.; Yunu, T. Utilization of mixed chicken waste for biodiesel production using single and combination of immobilized lipase as a catalyst. Biomass Convers. Bior. 2020, 1-14. [CrossRef]

200. Shahedi, M.; Yousefi, M.; Habibi, Z.; Mohammadi, M.; As'habi, M.A. Co-immobilization of Rhizomucor miehei lipase and Candida antarctica lipase B and optimization of biocatalytic biodiesel production from palm oil using response surface methodology. Renew. Energy 2019, 141, 847-857. [CrossRef]

201. Poppe, J.K.; Matte, C.R.; Fernandez-Lafuente, R.; Rodrigues, R.C.; Ayub, M.A.Z. Transesterification of waste frying oil and soybean oil by combi-lipases under ultrasound-assisted reactions. Appl. Biochem. Biotechnol. 2018, 186, 576-589. [CrossRef] [PubMed]

202. Ramos, M.D.; Miranda, L.P.; Fernandez-Lafuente, R.; Kopp, W.; Tardioli, P.W. Improving the yields and reaction rate in the ethanolysis of soybean oil by using mixtures of lipase CLEAs. Molecules 2019, 24, 4392. [CrossRef] [PubMed]

203. Lee, J.-H.; Lee, D.-H.; Lim, J.-S.; Um, B.-H.; Park, C.-H.; Kang, S.-W.; Kim, S.-W. Optimization of the process for biodiesel production using a mixture of immobilized Rhizopus oryzae and Candida rugosa lipases. J. Microbiol. Biotechnol. 2008, 18, 1927-1931.

204. Lee, J.H.; Kim, S.B.; Yoo, H.Y.; Lee, J.H.; Han, S.O.; Park, C.; Kim, S.W. Co-immobilization of Candida rugosa and Rhyzopus oryzae lipases and biodiesel production. Korean J. Chem. Eng. 2013, 30, 1335-1338. [CrossRef]

205. Lee, J.H.; Lee, J.H.; Kim, D.S.; Yoo, H.Y.; Park, C.; Kim, S.W. Biodiesel production by lipases co-immobilized on the functionalized activated carbon. Bioresour. Technol. Rep. 2019, 7, 100248. [CrossRef]

206. Toro, E.C.; Rodríguez, D.F.; Morales, N.; García, L.M.; Godoy, C.A. Novel combi-lipase systems for fatty acid ethyl esters production. Catalysts 2019, 9, 546. [CrossRef]

207. Gusniah, A.; Veny, H.; Hamzah, F. Ultrasonic assisted enzymatic transesterification for biodiesel production. Ind. Eng. Chem. Res. 2018, 58, 581-589. [CrossRef]

208. Kim, K.H.; Lee, O.K.; Lee, E.Y. Nano-immobilized biocatalysts for biodiesel production from renewable and sustainable resources. Catalysts 2018, 8, 68 .

209. Sohail, S.; Mumtaz, M.W.; Mukhtar, H.; Touqeer, T.; Anjum, M.K.; Rashid, U.; Wan Ab Karim Ghani, W.A.; Choong, T.S.Y. Spirogyra oil-based biodiesel: Response surface optimization of chemical and enzymatic transesterification and exhaust emission behavior. Catalysts 2020, 10, 1214. [CrossRef]

210. Lv, Y.; Sun, S.; Liu, J. Biodiesel production catalyzed by a methanol-tolerant lipase a from Candida antarctica in the presence of excess water. ACS Omega 2019, 4, 20064-20071. [CrossRef]

211. Lee, H.-s.; Lee, D.; Kim, S.; Kim, J. Effect of supercritical carbon dioxide on the enzymatic production of biodiesel from waste animal fat using immobilized Candida antarctica lipase B variant. BMC Biotechnol. 2017, 17, 1-6.

212. Gupta, A.R.; Rathod, V.K. Biodiesel synthesis from palm fatty acid distillate using enzyme immobilized on magnetic nanoparticles. SN Appl. Sci. 2020, 2, 1-10. [CrossRef]

213. Picó, E.A.; López, C.; Cruz-Izquierdo, Á.; Munarriz, M.; Iruretagoyena, F.J.; Serra, J.L.; Llama, M.J. Easy reuse of magnetic cross-linked enzyme aggregates of lipase B from Candida antarctica to obtain biodiesel from Chlorella vulgaris lipids. J. Biosci. Bioeng. 2018, 126, 451-457. [CrossRef]

214. Dill, L.P.; Kochepka, D.M.; Krieger, N.; Ramos, L.P. Synthesis of fatty acid ethyl esters with conventional and microwave heating systems using the free lipase B from Candida antarctica. Biocatal. Biotransform. 2019, 37, 25-34. [CrossRef]

215. Wang, L.; Liu, X.; Jiang, Y.; Liu, P.; Zhou, L.; Ma, L.; He, Y.; Li, H.; Gao, J. Silica nanoflowers-stabilized Pickering emulsion as a robust biocatalysis platform for enzymatic production of biodiesel. Catalysts 2019, 9, 1026. [CrossRef]

216. Makareviciene, V.; Gumbyte, M.; Sendzikiene, E. Simultaneous extraction of microalgae Ankistrodesmus sp. oil and enzymatic transesterification with ethanol in the mineral diesel medium. Food Bioprod. Process. 2019, 116, 89-97. [CrossRef] 
217. Santaraite, M.; Sendzikiene, E.; Makareviciene, V.; Kazancev, K. Biodiesel production by lipase-catalyzed in situ transesterification of rapeseed oil containing a high free fatty acid content with ethanol in diesel fuel media. Energies 2020, 13, 2588. [CrossRef]

218. Sun, S.; Li, K. Biodiesel production from phoenix tree seed oil catalyzed by liquid lipozyme TL100L. Renew. Energy 2020, 151, 152-160. [CrossRef]

219. Wancura, J.H.; Rosset, D.V.; Mazutti, M.A.; Ugalde, G.A.; de Oliveira, J.V.; Tres, M.V.; Jahn, S.L. Improving the soluble lipasecatalyzed biodiesel production through a two-step hydroesterification reaction system. Appl. Microbiol. Biotechnol. 2019, 103, 7805-7817. [CrossRef]

220. Rosset, D.V.; Wancura, J.H.; Ugalde, G.A.; Oliveira, J.V.; Tres, M.V.; Kuhn, R.C.; Jahn, S.L. Enzyme-catalyzed production of FAME by hydroesterification of soybean oil using the novel soluble lipase NS 40116. Appl. Biochem. Biotechnol. 2019, 188, 914-926. [CrossRef]

221. Malani, R.S.; Umriwad, S.B.; Kumar, K.; Goyal, A.; Moholkar, V.S. Ultrasound-assisted enzymatic biodiesel production using blended feedstock of non-edible oils: Kinetic analysis. Energy Convers. Manag. 2019, 188, 142-150. [CrossRef]

222. Rachmadona, N.; Amoah, J.; Quayson, E.; Hama, S.; Yoshida, A.; Kondo, A.; Ogino, C. Lipase-catalyzed ethanolysis for biodiesel production of untreated palm oil mill effluent. Sustain. Energy Fuels 2020, 4, 1105-1111. [CrossRef]

223. Kumar, D.; Das, T.; Giri, B.S.; Verma, B. Preparation and characterization of novel hybrid bio-support material immobilized from Pseudomonas cepacia lipase and its application to enhance biodiesel production. Renew. Energy 2020, 147, 11-24. [CrossRef]

224. Xie, W.; Huang, M. Fabrication of immobilized Candida rugosa lipase on magnetic $\mathrm{Fe}_{3} \mathrm{O}_{4}$-poly (glycidyl methacrylate-comethacrylic acid) composite as an efficient and recyclable biocatalyst for enzymatic production of biodiesel. Renew. Energy 2020, 158, 474-486. [CrossRef]

225. OstojčiĆ, M.; Budžaki, S.; Flanjak, I.; Rajs, B.B.; Barišić, I.; Tran, N.N.; Hessel, V.; Strelec, I. Production of biodiesel by Burkholderia cepacia lipase as a function of process parameters. Biotechnol. Prog. 2020. [CrossRef]

226. Badgujar, V.C.; Badgujar, K.C.; Yeole, P.M.; Bhanage, B.M. Enhanced biocatalytic activity of immobilized steapsin lipase in supercritical carbon dioxide for production of biodiesel using waste cooking oil. Bioprocess. Biosys. Eng. 2019, 42, 47-61. [CrossRef]

227. Shao, H.; Hu, X.; Sun, L.; Zhou, W. Gene cloning, expression in E. coli, and in vitro refolding of a lipase from Proteus sp. NH 2-2 and its application for biodiesel production. Biotechnol. Lett. 2019, 41, 159-169. [CrossRef]

228. Selvakumar, P.; Sivashanmugam, P. Ultrasound assisted oleaginous yeast lipid extraction and garbage lipase catalyzed transesterification for enhanced biodiesel production. Energy Convers. Manag. 2019, 179, 141-151. [CrossRef]

229. Wang, A.; Zhang, H.; Li, H.; Yang, S. Efficient production of methyl oleate using a biomass-based solid polymeric catalyst with high acid density. Adv. Polym. Technol. 2019, 2019. [CrossRef]

230. Lam, M.K.; Lee, K.T.; Mohamed, A.R. Homogeneous, heterogeneous and enzymatic catalysis for transesterification of high free fatty acid oil (waste cooking oil) to biodiesel: A review. Biotechnol. Adv. 2010, 28, 500-518. [CrossRef] [PubMed]

231. Gebremariam, S.N.; Marchetti, J.M. Techno-economic performance of a bio-refinery for the production of fuel-grade biofuel using a green catalyst. Biofuels Bioprod. Biorefin. 2019, 13, 936-949. [CrossRef]

232. Gebremariam, S.N.; Marchetti, J.M. Biodiesel production process using solid acid catalyst: Influence of market variables on the process's economic feasibility. Biofuels Bioprod. Biorefin. 2021, 15, 815-824. [CrossRef]

233. Gebremariam, S.; Marchetti, J. Techno-economic feasibility of producing biodiesel from acidic oil using sulfuric acid and calcium oxide as catalysts. Energy Convers. Manag. 2018, 171, 1712-1720. [CrossRef]

234. Kiss, F.E.; Jovanović, M.; Bošković, G.C. Economic and ecological aspects of biodiesel production over homogeneous and heterogeneous catalysts. Fuel Process. Technol. 2010, 91, 1316-1320. [CrossRef]

235. Gebremariam, S.N.; Marchetti, J.M. The effect of economic variables on a bio-refinery for biodiesel production using calcium oxide catalyst. Biofuels Bioprod. Biorefin. 2019, 13, 1333-1346. [CrossRef]

236. Gebremariam, S.; Marchetti, J. Economics of biodiesel production. Energy Convers. Manag. 2018, 168, 74-84. [CrossRef]

237. Jegannathan, K.R.; Eng-Seng, C.; Ravindra, P. Economic assessment of biodiesel production: Comparison of alkali and biocatalyst processes. Renew. Sustain. Energy Rev. 2011, 15, 745-751. [CrossRef] 